\title{
Theory of Continuous Rate-Dependent Hysteresis
}

\author{
Fayçal Ikhouane \\ Universitat Politècnica de Catalunya, Department of Mathematics. \\ Barcelona East School of Engineering, carrer Eduard Maristany, 16, 08019, Barcelona, Spain. \\ faycal.ikhouane@upc.edu
}

\begin{abstract}
Hysteresis is a special type of behavior ubiquitous in science and engineering: it consists in that slow inputs produce a loop in the steady-state part of the graph output-versus-input.

On the other hand, mathematical textbooks on hysteresis use a different property to define hysteresis processes: rate independence. This property says that the graph output-versus-input remains unchanged under a time-scale change.

However, experimental evidence shows the existence of physical processes that produce loops in steady state for slow inputs without being rate independent: these processes are called rate-dependent hysteresis.

This fact raises the following issue. How can we build a framework in which we can study hysteresis phenomena for which the rate-independence approximation is insufficient? The attempts to answer this question have been few and limited up till now.

In this paper we propose a mathematical framework for the description and analysis of rate-dependent hysteresis processes for which a continuous input produces a continuous output and a continuous hysteresis loop. The methodology that we use to obtain our theory consists in (1) making a list of experimentally observed properties of hysteresis which we call inferences, (2) proposing a mathematical equation -called premise- as a characteristic of hysteresis systems, and (3) proving analytically that the premise leads to all inferences. The operational formulation that we use provides a high degree of generality, and leads to several inferences from one single premise.

To illustrate the usefulness of the tools that we introduce, we propose a mathematical model that generates rate-dependent operators from rate-independent ones. We provide the analytic expression of the hysteresis loop of the rate-dependent operator in terms of the hysteresis loop of its rate-independent component. This result is illustrated by means of numerical simulations.
\end{abstract}

Keywords:

Rate dependent, Rate independent, Hysteresis 
PACS: 77.80.Dj, 75.60.-d

2000 MSC: 46T99, 93A99

\section{Introduction and problem statement}

A brief historical review of hysteresis. The word hysteresis was coined by Ewing in 1881, see Ref. [14]. In the period 1896-1902 Duhem published seven memoirs dedicated to the study of hysteresis, see Refs. [7]-[13]. Duhem considers a system subject to an external action that changes slowly so that the thermodynamical state of the system changes along a path of equilibria. The main characteristic of a hysteresis system is that these changes are not reversible.

During a large part of the twentieth century, "mathematical developments have been lagging behind those of physicists and engineers. Of course, mathematics has been used in works of applied scientists on hysteresis, but there it occurred more as calculus than in the form of functional analysis." [29, p. 12].

An important advance in the development of the mathematics of hysteresis is the formulation of rate independence by Bouc using the tools of functional analysis [5]. A translation into English of the part of Ref. [5] that deals with rate independence is provided in Ref. [18]. The mathematical formulation of rate independence is the following $[28$, p. 60]:

$$
\mathcal{H}\left(u \circ f, x_{0}\right)=\mathcal{H}\left(u, x_{0}\right) \circ f,
$$

in which $\mathcal{H}$ is a hysteresis operator, $u$ the input, $x_{0}$ the initial state, $f$ a time-scale change, and $\mathcal{H}\left(u, x_{0}\right)$ the output that corresponds to the input $u$ and initial state $x_{0}$.

More developments of the mathematics of hysteresis include the translation into English of the work of the mathematicians Krasnosel'skii and Pokrovskiǐ [21], the publication of a survey of models with hysteresis in 1993 [22] and of several monographs on hysteresis including Refs. [3, 4, 20, 23, 28].

Revisiting rate independence. In Equation (1) take $\mathcal{H}\left(u_{1}, x_{0}\right)=y_{1} ; u_{2}(t)=$ $u_{1}(2 t)$ that is $f(t)=2 t$; and $\mathcal{H}\left(u_{2}, x_{0}\right)=y_{2}$. This means that $y_{1}$ is the output that corresponds to the input $u_{1}$, and $y_{2}$ is the output that corresponds to the input $u_{2}$. Rate independence states that $y_{2}(t)=y_{1}(2 t)$ so that the graph $\left\{\left(u_{1}(t), y_{1}(t)\right), t \geq 0\right\}$ is exactly the same as $\left\{\left(u_{2}(t), y_{2}(t)\right), t \geq 0\right\}$. For rate independent systems, it does not matter whether the input varies slowly or not because the graph output-versus-input is the same for any change $f$ in time scale. For a rate-independent hysteresis, the graph output-versus-input $\left\{\left(u(t),\left[\mathcal{H}\left(u, x_{0}\right)\right](t)\right), t \geq 0\right\}$ is invariant under any change in time scale. 
Rate independence was used by Visintin in 1994 to define hysteresis systems: "Definition. Hysteresis = Rate Independent Memory Effect." [28, p. 13]. The memory-effect part of the definition excludes static nonlinearities which are rate independent but have no memory. Once we accept this definition, we are able to decide whether a particular mathematical model is a hysteresis or not. For example, by Visintin's definition, the following models are hysteresis models since they are rate independent and have a memory: the relay, Ishlinskiı̀'s model, Preisach's model, Duhem's model, Krasnosel'skiu and Pokrovskiı's hysteron and generalized play [22].

Rate independence is thus an attractive mathematical property. However, rate independence is but an approximation of the real behavior of hysteretic systems. Indeed, quoting from Visintin: "Usually rate-dependent effects get larger as the rate increases, and vanish as the rate vanishes. Therefore hysteresis is more evident at slow regimes, whereas it may be dominated by viscosity at fast regimes. In applications hysteresis appears only if the time-scale is sufficiently slow, and we are induced to regard as hysteretic those phenomena for which the rate-independent component prevails at typical regimes." [29, pp. 10-11].

Examples of references which consider hysteresis processes that are not rate independent include Refs. [1, 16, 30, 32] among others. This said, it is by no means obvious which property or properties are satisfied by real hysteresis rate-dependent systems. Even when such properties are detected, their description is generally qualitative.

Towards a definition of rate-dependent hysteresis. We consider that a definition or characterization of hysteresis is a set composed of three elements: a frame, a premise or a set of premises, and a set of inferences.

The frame refers to the general properties of the system, including the set of admissible inputs, outputs, and states.

An example of a frame is the following.

$(\mathscr{F} 1)$ Let $U, X, Y$ be arbitrary sets. Let $\mathcal{U}$ be the set of all functions $u: \mathbb{R}_{+} \rightarrow U, \mathcal{X}$ the set of all functions $x: \mathbb{R}_{+} \rightarrow X$, and $\mathcal{Y}$ the set of all functions $y: \mathbb{R}_{+} \rightarrow Y$. Then for any $\left(u, x_{0}\right) \in \mathcal{U} \times X$ we define $\mathcal{H}\left(u, x_{0}\right)=\mathcal{T} \circ\left[\Phi\left(u, x_{0}\right)\right]$ where $\Phi$ is a function (called operator in this work) $\Phi: \mathcal{U} \times X \rightarrow \mathcal{X}$, and $\mathcal{T}: X \rightarrow Y$ is a function, so that $\mathcal{H}: \mathcal{U} \times X \rightarrow \mathcal{Y}$.

The elements of $\mathcal{U}$ are called inputs, and the argument $t \in \mathbb{R}_{+}$of the input $u$ is called time. This means that the value of the input $u$ at time $t$ is $u(t)$.

The function $\mathcal{H}\left(u, x_{0}\right): \mathbb{R}_{+} \rightarrow Y$ is called the output that corresponds to the input $u \in \mathcal{U}$ and the initial state $x_{0} \in X$.

The function $\Phi\left(u, x_{0}\right): \mathbb{R}_{+} \rightarrow X$ is called the state that corresponds to the input 
$u \in \mathcal{U}$ and the initial state $x_{0} \in X$. If $\mathcal{T}$ is the identity function, then the state is also the output.

The premise or set of premises refers to a mathematical property or a set of mathematical properties that the system is supposed to satisfy under some special conditions.

An inference is a proposition reached by a process of deriving logical consequences from the assumed premises taking into account the frame.

The set of inferences that we are interested in consists of properties that are considered inherent to hysteresis systems. The discussion in [18, Section 2] provides the following inferences.

$(\mathscr{I} 1)$ When the input varies slowly, the system is approximately rate independent.

$(\mathscr{I} 2)$ The hysteresis loop is a steady-state mathematical object that involves an outputversus-input graph.

A hysteresis loop that is not a line is an indication that the system is a candidate for representing a hysteretic behavior.

$(\mathscr{I} 3)$ The hysteresis loop has a natural parametrization.

Indeed, the orientation of the hysteresis loop -clockwise or counterclockwise- is related to energy dissipation in some physical processes [6, Section 3.5]. If the hysteresis loop is just a set of points without a specification of the orientation, the information related to energy dissipation is lost.

The fourth inference comes from the observation made by Oh et al [25, p. 104] that to be step convergent is a necessary condition for a system to be hysteretic.

$(\mathscr{I} 4)$ The system is step convergent. This means that for any constant input and any initial state, the output converges to a finite constant as time goes to infinity.

The argument of Oh et al can be presented as follows. A necessary condition for a system to be a hysteresis is that it should have a graph output-versus-input in steady state for slowly varying inputs. The slowest input is a one that is constant since it does not vary. In steady state, that is when time approaches infinity, the corresponding output should approach some finite value in order to obtain a graph output-versus-input in steady state.

Aim of the paper. We seek and propose a frame as general as possible, with as few premises as possible, that lead to the set of inferences $\{(\mathscr{I} 1),(\mathscr{I} 2),(\mathscr{I} 3),(\mathscr{I} 4)\}$.

Novelty of the paper. To date, providing a mathematical definition or characterization for rate-dependent hysteresis has been attempted only in Refs [17] and [26]. None of these works leads to all inferences $(\mathscr{I} 1)-(\mathscr{I} 4)$.

The novelty of our work consists in the following items. 
(i) The methodology that we use to obtain our theory is sound: it consists (1) in making a list of the experimentally observed properties of hysteresis systems -that is the inferences-, (2) proposing a mathematical equation as a characteristic of hysteresis systems -this is the premise-, and (3) proving analytically that the premise leads to all inferences. To the best of our knowledge, this approach has not been attempted before in the case of rate-dependent hysteresis systems.

(ii) The characterization of rate-dependent hysteresis through the concept of confluence is new. The operational formulation that we use provides a high degree of generality, and leads to several inferences from one single premise.

(iii) We propose a new mathematical model that generates rate-dependent operators from rate-independent ones.

Organization of the paper.

The notation used throughout the paper is presented in Section 2. Different sets related to time-scale change are given in Section 3. The frame, premise and inferences are presented in Sections 4, 5, and 6 respectively. An application to the modeling of rate-dependent hysteresis is studied in Section 7 . Conclusions are provided in Section 8.

\section{Mathematical notation}

An ordered pair $a, b$ is denoted $(a, b)$ whilst the open interval $\{t \in \mathbb{R} \mid a<t<b\}$ is denoted $] a, b[$. The set of nonnegative integers is denoted $\mathbb{N}=\{0,1, \ldots\}$ and the set of nonnegative real numbers is denoted $\mathbb{R}_{+}=[0, \infty[$.

We say that a subset of $\mathbb{R}$ is measurable when it is Lebesgue measurable. Consider a function $g: I \subset \mathbb{R}_{+} \rightarrow \mathbb{R}^{n}$ where $n \in \mathbb{N} \backslash\{0\}$ and $I$ an interval. We say that $g$ is measurable when $g$ is $(M, B)$-measurable where $B$ is the class of Borel sets of $\mathbb{R}^{n}$, and $M$ is the class of measurable sets of $\mathbb{R}[31]$. For a measurable function $g: I \rightarrow \mathbb{R}^{n},\|g\|$ denotes the essential supremum of the function $|g|$ where $|\cdot|$ is the Euclidean norm on $\mathbb{R}^{n}$.

$C^{0}(I, J)$ denotes the set of continuous functions defined from the interval $I \subset \mathbb{R}_{+}$to the topological space $J$.

We consider the Sobolev space $W^{1 ; \infty}\left(\mathbb{R}_{+}, \mathbb{R}^{n}\right)$ of absolutely continuous functions $u$ : $\mathbb{R}_{+} \rightarrow \mathbb{R}^{n}$. For this class of functions, the derivative $\dot{u}$ is measurable, and we have $\|u\|<\infty,\|\dot{u}\|<\infty$. Endowed with the norm $\|u\|_{1, \infty}=\max (\|u\|,\|\dot{u}\|), W^{1 ; \infty}\left(\mathbb{R}_{+}, \mathbb{R}^{n}\right)$ is a Banach space [24, pp. 280-281].

Let $a \in \mathbb{Z}$ and $b \in \mathbb{R}$. Define the set $\llbracket a, \infty \llbracket=\{k \in \mathbb{Z} \mid k \geq a\}$. Let $f$ : $\llbracket a, \infty \llbracket \times] b, \infty[\rightarrow \mathbb{R}$. We say that

$$
\lim _{(k, \gamma) \rightarrow \infty} f(k, \gamma)=0
$$


if $\left.\forall \epsilon>0, \exists k_{\epsilon} \in \llbracket a, \infty \llbracket, \exists \gamma_{\epsilon} \in\right] b, \infty\left[\right.$ such that $\forall k \in \llbracket k_{\epsilon}, \infty \llbracket, \forall \gamma \in\left[\gamma_{\epsilon}, \infty[\right.$ we have $|f(k, \gamma)| \leq \epsilon$.

Let $a_{1}, a_{2} \in \mathbb{Z}$. Then we define the set $\llbracket a_{1}, a_{2} \rrbracket=\left\{k \in \mathbb{Z} \mid a_{1} \leq k \leq a_{2}\right\}$.

For any $\gamma \in] 0, \infty\left[\right.$ define the linear change is time scale $s_{\gamma}: \mathbb{R} \rightarrow \mathbb{R}$ by $s_{\gamma}(t)=$ $t / \gamma, \forall t \in \mathbb{R}$.

For any $a \in \mathbb{R}$ define the translation $\tau_{a}: \mathbb{R} \rightarrow \mathbb{R}$ by $\tau_{a}(t)=t+a, \forall t \in \mathbb{R}$.

A function $u \in C^{0}\left(\mathbb{R}_{+}, \mathbb{R}^{n}\right)$ is said to be $T$-periodic, where $\left.T \in\right] 0, \infty[$, if $u(t+T)=$ $u(t), \forall t \in \mathbb{R}_{+}$.

For any $T \in] 0, \infty\left[\right.$ we define $\Omega_{T}$ as the set of all $T$-periodic functions $u \in C^{0}\left(\mathbb{R}_{+}, \mathbb{R}^{n}\right)$, and $\Omega=\cup_{T \in] 0, \infty[} \Omega_{T}$.

\section{Time-scale change}

\subsection{The set $\mathbb{I}_{T ; \gamma}$}

Let $T \in] 0, \infty[$ and $\gamma \in] 0, \infty\left[\right.$. Let $f_{T ; \gamma} \in C^{0}\left(\mathbb{R}_{+}, \mathbb{R}_{+}\right)$be a function that satisfies (a) $-(\mathrm{c})$.

(a) $f_{T ; \gamma}$ is strictly increasing on $\mathbb{R}_{+}$.

(b) $f_{T ; \gamma}(0)=0, \lim _{t \rightarrow \infty} f_{T ; \gamma}(t)=\infty$, and $f_{T ; \gamma}(t+k \gamma T)=f_{T ; \gamma}(t)+k T, \forall t \in[0, \gamma T], \forall k \in$ $\mathbb{N}$.

(c) $f_{T ; \gamma}^{-1} \in C^{0}\left(\mathbb{R}_{+}, \mathbb{R}_{+}\right)$, and $f_{T ; \gamma}^{-1}(t+k T)=f_{T ; \gamma}^{-1}(t)+k \gamma T, \forall t \in[0, T], \forall k \in \mathbb{N}$.

The set of all such functions $f_{T ; \gamma}$ is denoted $\mathbb{I}_{T ; \gamma}$. An example of a function $f_{T ; \gamma}$ is given in Figure 1.

For any $\gamma \in] 0, \infty\left[\right.$ define the set $\mathbb{I}_{T ; 1} \circ s_{\gamma}=\left\{f_{T ; 1} \circ s_{\gamma}, f_{T ; 1} \in \mathbb{I}_{T ; 1}\right\}$.

Proposition 3.1. $\mathbb{I}_{T ; 1} \circ s_{\gamma}=\mathbb{I}_{T ; \gamma}$.

Proof. Straightforward.

Proposition 3.1 provides a practical way to construct the set $\mathbb{I}_{T ; \gamma}$ : pick all elements of $\mathbb{I}_{T ; 1}$ and compose each element with $s_{\gamma}$ to get all elements of $\mathbb{I}_{T ; \gamma}$.

For any $T \in] 0, \infty\left[\right.$ define the set $s_{\frac{1}{T}} \circ \mathbb{I}_{1 ; 1} \circ s_{T}=\left\{s_{\frac{1}{T}} \circ f_{1 ; 1} \circ s_{T}, f_{1 ; 1} \in \mathbb{I}_{1 ; 1}\right\}$.

Proposition 3.2. $s_{\frac{1}{T}} \circ \mathbb{I}_{1 ; 1} \circ s_{T}=\mathbb{I}_{T ; 1}$.

Proof. Straightforward.

Proposition 3.2 provides a practical way to construct the set $\mathbb{I}_{T ; 1}$ : pick all $f_{1 ; 1} \in \mathbb{I}_{1 ; 1}$ and compute $s_{\frac{1}{T}} \circ f_{1 ; 1} \circ s_{T}$ to get all elements of $\mathbb{I}_{T ; 1}$.

Corollary 3.1. $\mathbb{I}_{T ; \gamma}=s_{\frac{1}{T}} \circ \mathbb{I}_{1 ; 1} \circ s_{\gamma T}$.

Proof. Combine Propositions 3.1 and 3.2. 


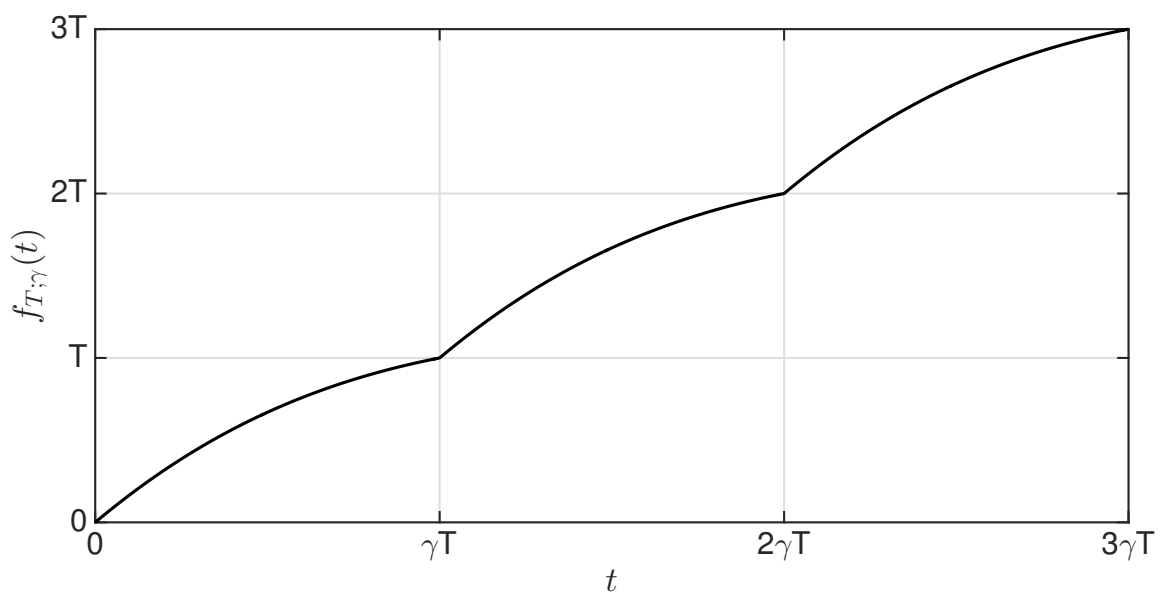

Figure 1: Example of a function $f_{T ; \gamma}$.

\section{The frame}

Let $\Xi$ be a Banach space with norm $|\cdot|$. Let $T \in] 0, \infty\left[\right.$, then $C^{0}([0, T], \Xi)$ is a Banach space with respect to the norm $\|\cdot\|$ defined by $\|a\|=\sup _{t \in[0, T]}|a(t)|$ for any $a \in C^{0}([0, T], \Xi)$. For any $w \in C^{0}\left(\mathbb{R}_{+}, \Xi\right)$ define $\|w\|=\sup _{t \in \mathbb{R}_{+}}|w(t)|$. Define the set $\mathcal{B}\left(\mathbb{R}_{+}, \Xi\right)=\left\{w \in C^{0}\left(\mathbb{R}_{+}, \Xi\right) \mid\|w\|<\infty\right\}$.

Let $n, m \in \mathbb{N} \backslash\{0\}$. We consider an operator $\Phi: C^{0}\left(\mathbb{R}_{+}, \mathbb{R}^{n}\right) \times \Xi \rightarrow C^{0}\left(\mathbb{R}_{+}, \Xi\right)$, an operator $\mathcal{H}: C^{0}\left(\mathbb{R}_{+}, \mathbb{R}^{n}\right) \times \Xi \rightarrow C^{0}\left(\mathbb{R}_{+}, \mathbb{R}^{m}\right)$, and a continuous function $\mathcal{T}: \Xi \rightarrow \mathbb{R}^{m}$ such that for any $\left(u, x_{0}\right) \in C^{0}\left(\mathbb{R}_{+}, \mathbb{R}^{n}\right) \times \Xi$ we have $\mathcal{H}\left(u, x_{0}\right)=\mathcal{T} \circ\left[\Phi\left(u, x_{0}\right)\right]$.

\section{The premise: confluence}

One of the main concepts of this paper is that of confluence. This concept will be presented formally in Section 5.2. In the following section 5.1, we present the concept of confluence in an informal way to make it easier to grasp for the reader.

\subsection{An informal introduction to the concept of confluence}

Consider the following system $y=\mathcal{H}\left(u, \xi_{0}\right)$ where

$$
\begin{aligned}
\dot{x}(t) & =\dot{u}(t)-|\dot{u}(t)| x(t), \\
\dot{y}(t) & =-y(t)+x(t), \\
x(0) & =x_{0}, \quad y(0)=y_{0}, \quad \xi_{0}=\left(x_{0}, y_{0}\right) .
\end{aligned}
$$




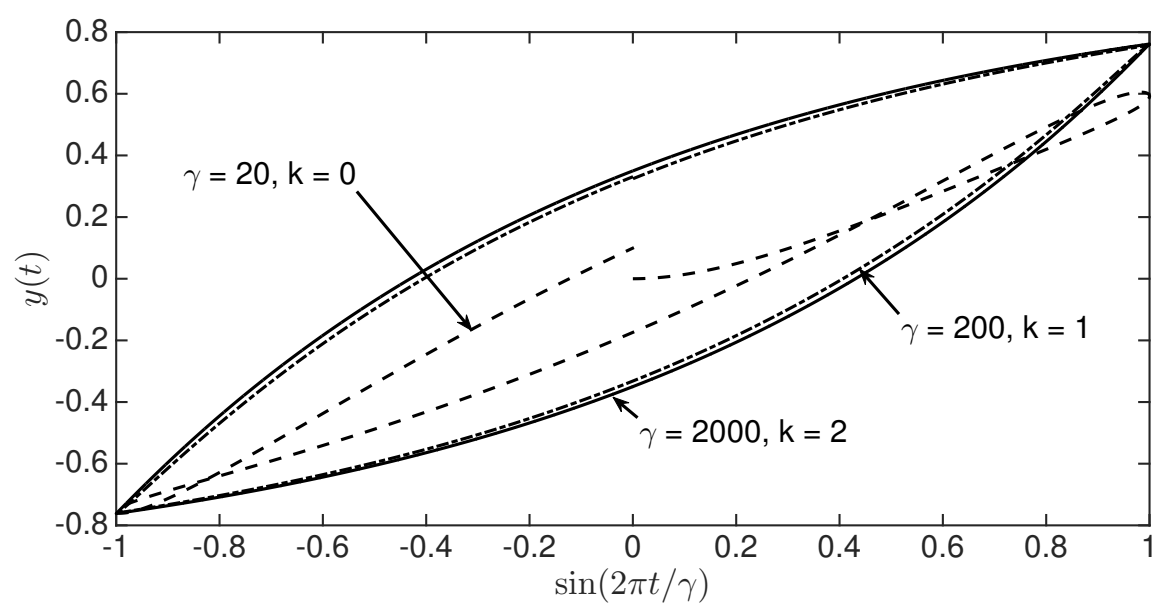

Figure 2: System (3), $y_{\gamma}(t)$ versus $u \circ s_{\gamma}(t)$ for $t \in[k \gamma T,(k+1) \gamma T]$.

When the input of the system is $u \circ s_{\gamma}(t)=\sin (2 \pi t / \gamma)$ where $u(t)=\sin (2 \pi t)$ and $s_{\gamma}(t)=$ $t / \gamma$, the output becomes $y_{\gamma}=\mathcal{H}\left(u \circ s_{\gamma}, \xi_{0}\right)$. With these notations (2) becomes

$$
\begin{aligned}
\dot{x}_{\gamma}(t) & =\overparen{u \circ s_{\gamma}}(t)-\left|\widetilde{\cdot \sim s_{\gamma}}(t)\right| x_{\gamma}(t), \\
\dot{y}_{\gamma}(t) & =-y_{\gamma}(t)+x_{\gamma}(t), \\
x_{\gamma}(0) & =x_{0}, \quad y_{\gamma}(0)=y_{0}, \quad \xi_{0}=\left(x_{0}, y_{0}\right) .
\end{aligned}
$$

The graph output-versus-input is the set $\left\{\left(u \circ s_{\gamma}(t), y_{\gamma}(t)\right), t \in \mathbb{R}_{+}\right\}$.

Inference $(\mathscr{I} 2)$ says that the hysteresis loop is a steady-state mathematical object that involves an output-versus-input graph. How can we define that object?

Observe that the function $u$ is periodic of period $T=1$ and $u \circ s_{\gamma}$ is periodic with period $\gamma T$. We see the domain $\mathbb{R}_{+}$of $u \circ s_{\gamma}$ as the union of all intervals of the form $[k \cdot \gamma T,(k+1) \cdot \gamma T]$ where $k=0,1,2, \ldots$ Let us consider increasing values of $\gamma$, say $\gamma=20, \gamma=200$, and $\gamma=2000$, and increasing values of $k$, say $k=0, k=1$, and $k=2$. Observe that larger and larger values of $\gamma$ correspond to a slower and slower inputs $u \circ s_{\gamma}$, whilst larger and larger values of $k$ correspond, in the interval $[k \cdot \gamma T,(k+1) \cdot \gamma T]$, to instants $t \geq k \cdot \gamma T$ for which the output gets closer and closer to the steady-state solution.

In Figure 2 we plot the graphs $A_{k, \gamma}=\left\{\left(u \circ s_{\gamma}(t), y_{\gamma}(t)\right), t \in[k \gamma T,(k+1) \gamma T]\right\}$ for the pairs $(k, \gamma)=(0,20),(k, \gamma)=(1,200)$, and $(k, \gamma)=(2,2000)$.

We observe that the graphs $A_{k, \gamma}$ converge to a closed curve as both $k$ and $\gamma$ increase. It would thus be tempting to use the convergence of the sets $A_{k, \gamma}$ with respect to the Hausdorff distance when both $k$ and $\gamma$ go to infinity as a definition for the hysteresis loop. 
However, this procedure would not lead to a natural parametrization of the hysteresis loop, that is to Inference $(\mathscr{I} 3)$.

Instead, let us consider the set $A_{k, \gamma}^{\prime}=\left\{\left(u(t), y_{\gamma} \circ s_{\frac{1}{\gamma}} \circ \tau_{k T}(t)\right), t \in[0, T]\right\}$ where $\tau_{k T}(t)=t+k T$. It can be checked that $A_{k, \gamma}^{\prime}=A_{k, \gamma}$.

Consider the parametrized curves $c_{1}:[0, T] \rightarrow \mathbb{R}^{2}$ defined by $c_{1}(t)=\left(u(t), y_{\gamma} \circ s_{\frac{1}{\gamma}} \circ\right.$ $\left.\tau_{k T}(t)\right)$, and $c_{2}:[k \gamma T,(k+1) \gamma T] \rightarrow \mathbb{R}^{2}$ defined by $c_{2}(t)=\left(u \circ s_{\gamma}(t), y_{\gamma}(t)\right)$. Take any point $(a, b) \in A_{k, \gamma}^{\prime}=A_{k, \gamma}$. The orientation of $c_{1}$ at $(a, b)$ is $\dot{c}_{1}\left(t_{1}\right) /\left|\dot{c}_{1}\left(t_{1}\right)\right|$ where $c_{1}\left(t_{1}\right)=(a, b)$. Also, the orientation of $c_{2}$ at $(a, b)$ is $\dot{c}_{2}\left(t_{2}\right) /\left|\dot{c}_{2}\left(t_{2}\right)\right|$ where $c_{2}\left(t_{2}\right)=(a, b)$. It can be checked that the parametrized curves $c_{1}$ and $c_{2}$ have the same orientation at $(a, b)$.

These considerations show that a definition of the hysteresis loop can be obtained in two steps.

The first step considers that the functions $\left.\left(y_{\gamma} \circ s_{\frac{1}{\gamma}} \circ \tau_{k T}\right)\right|_{[0, T]}$ converge in some sense to a function $\alpha:[0, T] \rightarrow \mathbb{R}$ when both $k$ and $\gamma$ go to infinity.

The second step considers the set $\mathcal{Q}=\{(u(t), \alpha(t)), t \in[0, T]\}$ as the hysteresis loop of $\mathcal{H}$. Then, $\mathcal{Q}$ has a natural parametrization inherited from the parametrization of the curve $c_{1}$ (or equivalently $c_{2}$ ).

What kind of convergence is needed? We need to make sure that this definition of the hysteresis loop is compatible with the convergence of the sets $A_{k, \gamma}$ to $\mathcal{G}$ with respect to the Hausdorff distance when both $k$ and $\gamma$ go to infinity. To this end, it is enough to ask for a uniform convergence on the time interval $[0, T]$ of the functions $y_{\gamma} \circ s_{\frac{1}{\gamma}} \circ \tau_{k T}$ to $\alpha$ (this is proved in Proposition 6.5).

To close this informal introduction, let us mention that the uniform convergence of the functions $\left.\left(\mathcal{H}\left(u \circ s_{\gamma}, \xi_{0}\right) \circ s_{\frac{1}{\gamma}} \circ \tau_{k T}\right)\right|_{[0, T]}$ to $\alpha$ is sufficient to obtain the following version of Inference $(\mathscr{I} 1)$ : when the input varies slowly, the system can be approximated by a process which is rate-independent with respect to linear changes in time scale (this is proved in Proposition 6.3).

However, our aim is to obtain a stronger version of Inference $(\mathscr{I} 1)$ which states that when the input varies slowly, the system can be approximated by a process which is rate-independent with respect to changes in time scale that can be linear or nonlinear. This is why we consider a -possibly nonlinear- change in time scale $f_{T ; \gamma}$ in Section 3, and confluence is defined as the uniform convergence of the functions $\left(\mathcal{H}\left(u \circ f_{T ; \gamma}, \xi_{0}\right) \circ f_{T ; \gamma}^{-1} \circ\right.$ $\left.\tau_{k T}\right)\left.\right|_{[0, T]}$ when both $k$ and $\gamma$ go to infinity. 


\subsection{The formal definition of confluence}

Let $\mathbb{S}$ be a set such that Id $\in \mathbb{S} \subset \mathbb{I}_{1 ; 1}$ where Id $: \mathbb{R}_{+} \rightarrow \mathbb{R}_{+}$is the identity function defined by $\operatorname{Id}(t)=t, \forall t \in \mathbb{R}_{+}$. For any $\left.T, \gamma \in\right] 0, \infty[$ define the set

$$
\mathbb{S}_{T ; \gamma}=\left\{f_{T ; \gamma}=s_{\frac{1}{T}} \circ f_{1 ; 1} \circ s_{\gamma T}, f_{1 ; 1} \in \mathbb{S}\right\}=s_{\frac{1}{T}} \circ \mathbb{S} \circ s_{\gamma T}
$$

Then $s_{\gamma} \in \mathbb{S}_{T ; \gamma} \subset \mathbb{I}_{T ; \gamma}$.

Definition 5.1. Let $\left.\left(T, u, x_{0}\right) \in\right] 0, \infty\left[\times \Omega_{T} \times \Xi\right.$. We say that the operator $\Phi$ is confluent with respect to $\left(u, x_{0}, \mathbb{S}\right)$ if there exists a function $\alpha_{u ; \Phi ; x_{0} ; \mathbb{S}} \in C^{0}([0, T], \Xi)$ such that the following holds. Define the function $\left.\wp_{\Phi}: \mathbb{N} \times\right] 0, \infty\left[\rightarrow \mathbb{R}_{+} \cup\{\infty\}\right.$ by

$$
\forall(k, \gamma) \in \mathbb{N} \times] 0, \infty\left[, \wp_{\Phi}(k, \gamma)=\sup _{f_{T ; \gamma} \in \mathbb{S}_{T ; \gamma}}\left\|\left.\left(\Phi\left(u \circ f_{T ; \gamma}, x_{0}\right) \circ f_{T ; \gamma}^{-1} \circ \tau_{k T}\right)\right|_{[0, T]}-\alpha_{u ; \Phi ; x_{0} ; \mathbb{S}}\right\|\right.
$$

Then,

$$
\lim _{(k, \gamma) \rightarrow \infty} \wp_{\Phi}(k, \gamma)=0
$$

Proposition 5.1. The function $\alpha_{u ; \Phi ; x_{0} ; \mathbb{S}}$ does not depend on the set $\mathbb{S}$.

Proof. Take two sets $\mathbb{S}$ and $\mathbb{S}^{\prime}$ such that $\Phi$ is confluent with respect to $\left(u, x_{0}, \mathbb{S}\right)$ and $\left(u, x_{0}, \mathbb{S}^{\prime}\right)$. Since $\operatorname{Id} \in \mathbb{S} \cap \mathbb{S}^{\prime}$ it comes that $\alpha_{u ; \Phi ; x_{0} ; \mathbb{S}}=\alpha_{u ; \Phi ; x_{0} ;\{\operatorname{Id}\}}=\alpha_{u ; \Phi ; x_{0} ; \mathbb{S}^{\prime}}$

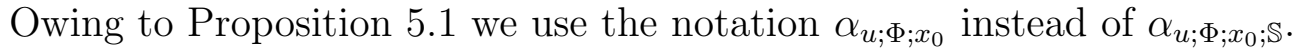

When $\Phi$ is confluent with respect to $\left(u, x_{0}, \mathbb{S}\right)$, the set

$$
\mathcal{Q}_{u ; \Phi ; x_{0}}=\left\{\left(u(t), \alpha_{u ; \Phi ; x_{0}}(t)\right), t \in[0, T]\right\}
$$

is called the hysteresis loop of the operator $\Phi$ with respect to $\left(u, x_{0}\right)$.

A similar definition can be made for the operator $\mathcal{H}$.

Definition 5.2. Let $\left.\left(T, u, x_{0}\right) \in\right] 0, \infty\left[\times \Omega_{T} \times \Xi\right.$. We say that the operator $\mathcal{H}$ is confluent with respect to $\left(u, x_{0}, \mathbb{S}\right)$ if there exists a function $\alpha_{u ; \mathcal{H} ; x_{0}} \in C^{0}\left([0, T], \mathbb{R}^{m}\right)$ such that the following holds. Define the function $\left.\wp_{\mathcal{H}}: \mathbb{N} \times\right] 0, \infty\left[\rightarrow \mathbb{R}_{+} \cup\{\infty\}\right.$ by

$$
\forall(k, \gamma) \in \mathbb{N} \times] 0, \infty\left[, \wp_{\mathcal{H}}(k, \gamma)=\sup _{f_{T ; \gamma} \in \mathbb{S}_{T ; \gamma}}\left\|\left.\left(\mathcal{H}\left(u \circ f_{T ; \gamma}, x_{0}\right) \circ f_{T ; \gamma}^{-1} \circ \tau_{k T}\right)\right|_{[0, T]}-\alpha_{u ; \mathcal{H} ; x_{0}}\right\|\right.
$$

Then,

$$
\lim _{(k, \gamma) \rightarrow \infty} \wp_{\mathcal{H}}(k, \gamma)=0
$$


If $\mathcal{H}$ is confluent with respect to $\left(u, x_{0}, \mathbb{S}\right)$, the set

$$
\mathcal{Q}_{u ; \mathcal{H} ; x_{0}}=\left\{\left(u(t), \alpha_{u ; \mathcal{H} ; x_{0}}(t)\right), t \in[0, T]\right\}
$$

is called the hysteresis loop of the operator $\mathcal{H}$ with respect to $\left(u, x_{0}\right)$.

Proposition 5.2. If $\Phi$ is confluent with respect to $\left(u, x_{0}, \mathbb{S}\right)$ then $\mathcal{H}$ is confluent with respect to $\left(u, x_{0}, \mathbb{S}\right)$ and $\alpha_{u ; \mathcal{H} ; x_{0}}=\mathcal{T} \circ \alpha_{u ; \Phi ; x_{0}}$.

Proof. Since $\mathcal{T}$ is continuous, it is uniformly continuous on the closed ball $\overline{\mathscr{B}}\left(\mathbf{0}_{\Xi}, r\right)$ with center $\mathbf{0}_{\Xi} \in \Xi$ and radius $r=1+\left\|\alpha_{u ; \Phi ; x_{0}}\right\|$. Take $\left.\epsilon \in\right] 0, \infty\left[\right.$, then $\left.\left.\exists \delta_{\epsilon} \in\right] 0,1\right]$ such that $|\mathcal{T}(\boldsymbol{x})-\mathcal{T}(\boldsymbol{y})| \leq \epsilon, \forall \boldsymbol{x}, \boldsymbol{y} \in \overline{\mathscr{B}}\left(\mathbf{0}_{\Xi}, r\right)$ with $|\boldsymbol{x}-\boldsymbol{y}| \leq \delta_{\epsilon}$. From Equations (4)-(5) it comes that $\left.\exists k_{\epsilon} \in \mathbb{N}, \exists \gamma_{\epsilon} \in\right] 0, \infty\left[\right.$ such that $\forall k \in \llbracket k_{\epsilon}, \infty \llbracket, \forall \gamma \in\left[\gamma_{\epsilon}, \infty[\right.$ we have

$$
\sup _{f_{T ; \gamma} \in \mathbb{S}_{T ; \gamma}}\left\|\left.\left(\Phi\left(u \circ f_{T ; \gamma}, x_{0}\right) \circ f_{T ; \gamma}^{-1} \circ \tau_{k T}\right)\right|_{[0, T]}-\alpha_{u ; \Phi ; x_{0}}\right\| \leq \delta_{\epsilon} .
$$

Equation (10) implies that $\forall k \in \llbracket k_{\epsilon}, \infty \llbracket, \forall \gamma \in\left[\gamma_{\epsilon}, \infty[\right.$,

$$
\sup _{f_{T ; \gamma} \in \mathbb{S}_{T ; \gamma}}\left\|\left.\left(\Phi\left(u \circ f_{T ; \gamma}, x_{0}\right) \circ f_{T ; \gamma}^{-1} \circ \tau_{k T}\right)\right|_{[0, T]}\right\| \leq\left\|\alpha_{u ; \Phi ; x_{0}}\right\|+\delta_{\epsilon} \leq r .
$$

Equation (11) shows that $\forall t \in[0, T], \forall k \in \llbracket k_{\epsilon}, \infty \llbracket, \forall \gamma \in\left[\gamma_{\epsilon}, \infty[\right.$ we have

$$
\left.\left(\Phi\left(u \circ f_{T ; \gamma}, x_{0}\right) \circ f_{T ; \gamma}^{-1} \circ \tau_{k T}\right)\right|_{[0, T]}(t) \in \overline{\mathscr{B}}\left(\mathbf{0}_{\Xi}, r\right) \text { and } \alpha_{u ; \Phi ; x_{0}}(t) \in \overline{\mathscr{B}}\left(\mathbf{0}_{\Xi}, r\right)
$$

which, combined with (10), shows that $\forall k \in \llbracket k_{\epsilon}, \infty \llbracket, \forall \gamma \in\left[\gamma_{\epsilon}, \infty[\right.$,

$$
\sup _{f_{T ; \gamma} \in \mathbb{S}_{T ; \gamma}} \| \underbrace{\mathcal{T} \circ\left(\Phi\left(u \circ f_{T ; \gamma}, x_{0}\right)\right.}_{\mathcal{H}\left(u \circ f_{T ; \gamma}, x_{0}\right)} \circ f_{T ; \gamma}^{-1} \circ \tau_{k T})\left.\right|_{[0, T]}-\underbrace{\mathcal{T} \circ \alpha_{u ; \Phi ; x_{0}}}_{\alpha_{u ; ; ; ;}} \| \leq \epsilon .
$$

Proposition 5.2 says that $\mathcal{H}$ is confluent whenever $\Phi$ is confluent. This is why we focus on the confluence of $\Phi$ in this paper.

\section{The inferences}

In this section we obtain the set of inferences $\{(\mathscr{I} 1),(\mathscr{I} 2),(\mathscr{I} 3),(\mathscr{I} 4)\}$. We first start by observing that for large enough $\gamma$, the output $\Phi\left(u \circ f_{T ; \gamma}, x_{0}\right)$ is always bounded. 
Proposition 6.1. Let $T \in] 0, \infty\left[, u \in \Omega_{T}\right.$, and $x_{0} \in \Xi$. If the operator $\Phi$ is confluent with respect to $\left(u, x_{0}, \mathbb{S}\right)$ then there exists $\left.\gamma_{u ; x_{0}} \in\right] 0, \infty\left[\right.$ such that $\forall \gamma \geq \gamma_{u ; x_{0}}$ we have the following: $\forall f_{T ; \gamma} \in \mathbb{S}_{T ; \gamma}, \Phi\left(u \circ f_{T ; \gamma}, x_{0}\right) \in \mathcal{B}\left(\mathbb{R}_{+}, \Xi\right)$. In particular $\forall \gamma \geq \gamma_{u ; x_{0}}, \Phi\left(u \circ s_{\gamma}, x_{0}\right) \in$ $\mathcal{B}\left(\mathbb{R}_{+}, \Xi\right)$.

Proof. From Definition 5.1 it comes that $\exists \alpha_{u ; \Phi ; x_{0}} \in C^{0}([0, T], \Xi)$ such that we have the following: $\left.\exists \gamma_{u ; x_{0}} \in\right] 0, \infty\left[\right.$ and $\exists k_{u ; x_{0}} \in \mathbb{N} \backslash\{0\}$ such that $\forall \gamma \in\left[\gamma_{u ; x_{0}}, \infty[\right.$ and $\forall k \in$ $\llbracket k_{u ; x_{0}}, \infty \llbracket$ we have

$$
\sup _{f_{T ; \gamma} \in \mathbb{S}_{T ; \gamma}}\left\|\left.\left(\Phi\left(u \circ f_{T ; \gamma}, x_{0}\right) \circ f_{T ; \gamma}^{-1} \circ \tau_{k T}\right)\right|_{[0, T]}-\alpha_{u ; \Phi ; x_{0}}\right\| \leq 1,
$$

so that $\forall \gamma \in\left[\gamma_{u ; x_{0}}, \infty[\right.$ we get

$$
\sup _{f_{T ; \gamma} \in \mathbb{S}_{T ; \gamma}}\left\|\left.\left(\Phi\left(u \circ f_{T ; \gamma}, x_{0}\right)\right)\right|_{\left[k_{u ; x_{0}} \gamma T, \infty[\right.}\right\| \leq 1+\left\|\alpha_{u ; \Phi ; x_{0}}\right\| .
$$

The proposition follows from Equation (14) along with the fact that $\Phi\left(u \circ f_{T ; \gamma}, x_{0}\right)$ is continuous.

\subsection{Inference $\left(\mathscr{I}_{4}\right)$}

Definition 6.1. We say that $\Phi$ is step convergent if $\forall\left(u_{0}, x_{0}\right) \in \mathbb{R}^{n} \times \Xi$ the following holds. Define $\mathbf{u}_{0} \in C^{0}\left(\mathbb{R}_{+}, \mathbb{R}^{n}\right)$ by $\mathbf{u}_{0}(t)=u_{0}, \forall t \in \mathbb{R}_{+}$. Then, there exists $\ell \in \Xi$ such that $\lim _{t \rightarrow \infty}\left[\Phi\left(\mathbf{u}_{0}, x_{0}\right)\right](t)=\ell$.

Proposition 6.2. Let $u_{0} \in \mathbb{R}^{n}$ and $x_{0} \in \Xi$. Define $\mathbf{u}_{0} \in \Omega_{T=1}$ by $\mathbf{u}_{0}(t)=u_{0}, \forall t \in \mathbb{R}_{+}$ (observe that the function $\mathbf{u}_{0}$ can be considered 1-periodic). If the operator $\Phi$ is confluent with respect to $\left(\mathbf{u}_{0}, x_{0}, \mathbb{S}\right)$ then there exists $\ell \in \Xi$ such that $\lim _{t \rightarrow \infty}\left[\Phi\left(\mathbf{u}_{0}, x_{0}\right)\right](t)=\ell$. Moreover, $\forall t \in[0,1], \alpha_{\mathbf{u}_{0} ; \Phi ; x_{0}}(t)=\ell$.

Proof. From Definition 5.1 we can find a function $\alpha_{\mathbf{u}_{0} ; \Phi ; x_{0}} \in C^{0}([0,1], \Xi)$ such that the following holds: $\left.\forall \epsilon>0, \exists \gamma_{\epsilon} \in\right] 0, \infty\left[\right.$ and $\exists k_{\epsilon} \in \mathbb{N} \backslash\{0\}$ such that $\forall \gamma \geq \gamma_{\epsilon}$ and $\forall k \geq k_{\epsilon}$ we have

$$
\left\|\left.\left(\Phi\left(\mathbf{u}_{0}, x_{0}\right) \circ s_{\frac{1}{\gamma}} \circ \tau_{k}\right)\right|_{[0,1]}-\alpha_{\mathbf{u}_{0} ; \Phi ; x_{0}}\right\| \leq \epsilon .
$$

Owing to the continuity of $\Phi\left(\mathbf{u}_{0}, x_{0}\right)$ and $\alpha_{\mathbf{u}_{0} ; \Phi ; x_{0}}$, Equation (15) implies that

$$
\left|\left[\Phi\left(\mathbf{u}_{0}, x_{0}\right)\right]\left(\gamma k_{\epsilon}\right)-\alpha_{\mathbf{u}_{0} ; \Phi ; x_{0}}(0)\right| \leq \epsilon, \forall \gamma \geq \gamma_{\epsilon}
$$

which ends the proof by taking $\ell=\alpha_{\mathbf{u}_{0} ; \Phi ; x_{0}}(0)$. The last part of the assertion follows from taking $t$ as argument in Equation (15).

Proposition 6.2 says that a confluent operator is also step convergent. Thus confluence leads to Inference $(\mathscr{I} 4)$. 


\subsection{Inference ( $\mathscr{I} 1)$}

In what follows we analyze the relationship between confluence and Inference $(\mathscr{I} 1)$. To this end we first prove that the hysteresis loop is a closed curve (Proposition 6.3). This fact will allow us to construct a rate-independent operator that approximates the operator $\Phi$ in steady state when the input is periodic and varies slowly (Proposition 6.4 and Theorem 6.1).

Proposition 6.3. Let $T \in] 0, \infty\left[, u \in \Omega_{T}\right.$ and $x_{0} \in \Xi$. Suppose that the operator $\Phi$ is confluent with respect to $\left(u, x_{0}, \mathbb{S}\right)$. Then $\alpha_{u ; \Phi ; x_{0}}(0)=\alpha_{u ; \Phi ; x_{0}}(T)$.

Proof. Let $\epsilon \in] 0, \infty\left[\right.$. Then there exist $\left.\gamma_{\epsilon} \in\right] 0, \infty\left[\right.$ and $k_{\epsilon} \in \mathbb{N}$ such that $\forall \gamma \in\left[\gamma_{\epsilon}, \infty[\right.$ and $\forall k \in \llbracket k_{\epsilon}, \infty \llbracket$ we have

$$
\left\|\left.\left(\Phi\left(u \circ s_{\gamma}, x_{0}\right) \circ s_{\frac{1}{\gamma}} \circ \tau_{k T}\right)\right|_{[0, T]}-\alpha_{u ; \Phi ; x_{0}}\right\| \leq \epsilon .
$$

Define $\eta_{\epsilon}=\left\lceil\max \left(\gamma_{\epsilon}, k_{\epsilon}\right)\right\rceil$ where $\lceil\cdot\rceil$ refers to the ceiling function. Let $k \in \llbracket \eta_{\epsilon}, \infty \llbracket$, then (17) gives

$$
\begin{array}{r}
\left\|\left.\left(\Phi\left(u \circ s_{k}, x_{0}\right) \circ s_{\frac{1}{k}} \circ \tau_{k T}\right)\right|_{[0, T]}-\alpha_{u ; \Phi ; x_{0}}\right\| \leq \epsilon, \\
\left\|\left.\left(\Phi\left(u \circ s_{k+1}, x_{0}\right) \circ s_{\frac{1}{k+1}} \circ \tau_{k T}\right)\right|_{[0, T]}-\alpha_{u ; \Phi ; x_{0}}\right\| \leq \epsilon .
\end{array}
$$

Equation (18) gives $\left|\left[\Phi\left(u \circ s_{k}, x_{0}\right)\right]\left(k^{2} T\right)-\alpha_{u ; \Phi ; x_{0}}(0)\right| \leq \epsilon$ which implies that the sequence $\mathbf{s}_{1}=\left\{\left[\Phi\left(u \circ s_{k}, x_{0}\right)\right]\left(k^{2} T\right)\right\}_{k \in \mathbb{N} \backslash\{0\}}$ converges in $\Xi$ to $\alpha_{u ; \Phi ; x_{0}}(0)$.

Equation (19) provides $\left|\left[\Phi\left(u \circ s_{k+1}, x_{0}\right)\right]\left((k+1)^{2} T\right)-\alpha_{u ; \Phi ; x_{0}}(T)\right| \leq \epsilon$ which implies that the sequence $\mathbf{s}_{2}=\left\{\left[\Phi\left(u \circ s_{k+1}, x_{0}\right)\right]\left((k+1)^{2} T\right)\right\}_{k \in \mathbb{N}}$ converges in $\Xi$ to $\alpha_{u ; \Phi ; x_{0}}(T)$.

The proposition follows from that fact that $\mathbf{s}_{1}=\mathbf{s}_{2}$.

Proposition 6.3 says that the hysteresis loop $\mathcal{Q}_{u ; \Phi ; x_{0}}$ is a closed curve.

Definition 6.2. Let $\mathscr{U} \subset C^{0}\left(\mathbb{R}_{+}, \mathbb{R}^{n}\right)$, $\mathscr{W}$ a set of homeomorphisms of $\mathbb{R}_{+}$, and $\mathscr{X} \subset \Xi$. We say that $\Phi$ is $(\mathscr{U}, \mathscr{W}, \mathscr{X})$-rate independent if $\forall u \in \mathscr{U}, \forall f \in \mathscr{W}, \forall x_{0} \in \mathscr{X}$ we have $\Phi\left(u \circ f, x_{0}\right)=\Phi\left(u, x_{0}\right) \circ f$.

Observe that if $\Phi$ is $(\mathscr{U}, \mathscr{W}, \mathscr{X})$-rate independent then $\mathcal{H}$ is also $(\mathscr{U}, \mathscr{W}, \mathscr{X})$-rate independent.

Theorem 6.1. The statements (a) and (b) are equivalent. 
(a) The operator $\Phi$ is confluent with respect to $\left(u, x_{0}, \mathbb{S}\right)$ for all $\left(u, x_{0}\right) \in \Omega \times \Xi$.

(b) There exist unique operators $\Phi^{\circ}: \Omega \times \Xi \rightarrow \Omega$ and $\Phi^{\ddagger}: \Omega \times \Xi \rightarrow C^{0}\left(\mathbb{R}_{+}, \Xi\right)$ such that (i)-(iii) hold.

(i) $\Phi=\Phi^{\circ}+\Phi^{\ddagger}$.

(ii) $\forall T \in] 0, \infty\left[\right.$, $\Phi^{\circ}$ is $\left(\Omega_{T}, \bigcup_{\gamma \in] 0, \infty[} \mathbb{S}_{T ; \gamma}, \Xi\right)$-rate independent.

(iii) Let $T \in] 0, \infty\left[\right.$ be arbitrary. For all $u \in \Omega_{T}$ and all $x_{0} \in \Xi$ we have

(iii-1) $\Phi^{\circ}\left(u, x_{0}\right)$ is $T$-periodic.

(iii-2) $\lim _{(k, \gamma) \rightarrow \infty}\left(\sup _{f_{T ; \gamma} \in \mathbb{S}_{T ; \gamma}}\left\|\left.\Phi^{\ddagger}\left(u \circ f_{T ; \gamma}, x_{0}\right)\right|_{[k \gamma T, \infty[}\right\|\right)=0$.

Proof. (a) $\Rightarrow$ (b).

Existence. Define the operator $\Phi^{\circ}: \Omega \times \Xi \rightarrow \Omega$ by

$$
\left[\Phi^{\circ}\left(u, x_{0}\right)\right](t+k T)=\alpha_{u ; \Phi ; x_{0}}(t), \forall t \in[0, T], \forall k \in \mathbb{N},
$$

where $T \in] 0, \infty\left[, u \in \Omega_{T}\right.$, and $x_{0} \in \Xi$.

Defining $\Phi^{\ddagger}=\Phi-\Phi^{\circ}$ gives (i). Then (iii-1) follows by construction of $\Phi^{\circ}$. To prove (ii) we proceed as follows.

Let $\lambda \in] 0, \infty\left[\right.$ and $f_{T ; \lambda} \in \mathbb{S}_{T ; \lambda}$ be given, then from Equation (5) we get

$$
\lim _{(k, \gamma) \rightarrow \infty}\left\|\left.(\Phi(u \circ \underbrace{f_{T ; \lambda} \circ s_{\frac{\gamma}{\lambda}}}_{f_{T ; \gamma}}, x_{0}) \circ \underbrace{s_{\frac{\lambda}{\gamma}} \circ f_{T ; \lambda}^{-1}}_{f_{T ; \gamma}^{-1}} \circ \tau_{k T})\right|_{[0, T]}-\alpha_{u ; \Phi ; x_{0}}\right\|=0 .
$$

On the other hand, $u \circ f_{T ; \lambda} \in \Omega_{\lambda T}$ so that

$$
\lim _{\left(k, \gamma^{\prime}\right) \rightarrow \infty}\left\|\left.\left(\Phi\left(u \circ f_{T ; \lambda} \circ s_{\gamma^{\prime}}, x_{0}\right) \circ s_{\frac{1}{\gamma^{\prime}}} \circ \tau_{k \cdot \lambda T}\right)\right|_{[0, \lambda T]}-\alpha_{u \circ f_{T ; \lambda} ; \Phi ; x_{0}}\right\|=0 .
$$

Observe that $\left.\left(f_{T ; \lambda}^{-1} \circ \tau_{k T}\right)\right|_{[0, T]}=\left.\left(\tau_{k \cdot \lambda T} \circ f_{T ; \lambda}^{-1}\right)\right|_{[0, T]}$. Taking $\gamma^{\prime}=\gamma / \lambda$ and combining Equations (20) and (21) it comes that $\alpha_{u \circ f_{T ; \lambda} ; \Phi ; x_{0}} \circ f_{T ; \lambda}^{-1}=\alpha_{u ; \Phi ; x_{0}}$ which gives (ii).

To get (iii-2) write $\Phi=\Phi^{\circ}+\Phi^{\ddagger}$ in Equation (5), then using (ii) and (iii-1) it comes that

$$
\lim _{(k, \gamma) \rightarrow \infty}\left(\sup _{f_{T ; \gamma} \in \mathbb{S}_{T ; \gamma}}\left\|\left.\left(\Phi^{\ddagger}\left(u \circ f_{T ; \gamma}, x_{0}\right) \circ f_{T ; \gamma}^{-1} \circ \tau_{k T}\right)\right|_{[0, T]}\right\|\right)=0 .
$$


Take $\epsilon \in] 0, \infty\left[\right.$, then $\left.\exists k_{\epsilon} \in \mathbb{N}, \exists \gamma_{\epsilon} \in\right] 0, \infty\left[\right.$ such that $\forall k \in \llbracket k_{\epsilon}, \infty \llbracket, \forall \gamma \in\left[\gamma_{\epsilon}, \infty[\right.$ we have

$$
\sup _{f_{T ; \gamma} \in \mathbb{S}_{T ; \gamma}}\left\|\left.\left(\Phi^{\ddagger}\left(u \circ f_{T ; \gamma}, x_{0}\right) \circ f_{T ; \gamma}^{-1} \circ \tau_{k T}\right)\right|_{[0, T]}\right\| \leq \epsilon,
$$

so that

$$
\sup _{f_{T ; \gamma} \in \mathbb{S}_{T ; \gamma}}\left\|\left.\left(\Phi^{\ddagger}\left(u \circ f_{T ; \gamma}, x_{0}\right) \circ f_{T ; \gamma}^{-1}\right)\right|_{\left[k_{\epsilon} T, \infty[\right.}\right\| \leq \epsilon .
$$

Then (iii-2) comes from the fact that $f_{T ; \gamma}^{-1}\left(k_{\epsilon} T\right)=k_{\epsilon} \gamma T$.

Uniqueness. Suppose that (i)-(iii) hold. Then (iii-2) $\Leftrightarrow(24) \Leftrightarrow(23) \Leftrightarrow(22)$. Using (i) we obtain $\Phi^{\ddagger}=\Phi-\Phi^{\circ}$ so that substituting into (22) and using (ii)-(iii-1) we get

$$
\lim _{(k, \gamma) \rightarrow \infty}\left(\sup _{f_{T ; \gamma} \in \mathbb{S}_{T ; \gamma}}\left\|\left.\left(\Phi\left(u \circ f_{T ; \gamma}, x_{0}\right) \circ f_{T ; \gamma}^{-1} \circ \tau_{k T}\right)\right|_{[0, T]}-\Phi^{\circ}\left(u, x_{0}\right)\right\|\right)=0
$$

which shows that we have necessarily $\left[\Phi^{\circ}\left(u, x_{0}\right)\right](t)=\alpha_{u ; \Phi ; x_{0}}(t), \forall t \in[0, T]$.

$(\mathrm{b}) \Rightarrow(\mathrm{a})$.

The proof is similar to that of uniqueness in $(a) \Rightarrow(b)$.

Theorem 6.1 (iii-2) says that far from the transient, that is for $t \geq k \cdot \gamma T$ with a large $k$, the term $\Phi^{\ddagger}$ becomes small when the input $u \circ f_{T ; \gamma}$ varies slowly, which happens for a large $\gamma$. In this situation, (i) says that $\Phi$ can be approximated by $\Phi^{\circ}$ which is a rate-independent operator by (ii). This is precisely Inference $(\mathscr{I} 1)$.

We call canonical decomposition the equality (i) that gives the operator $\Phi$ as the sum of a rate-independent operator $\Phi^{\circ}$ and an operator $\Phi^{\ddagger}$ that vanishes in steady state for slowly varying inputs. The operator $\Phi^{\circ}$ is called the rate-independent component of $\Phi$, and $\Phi^{\ddagger}$ the nonhysteretic component of $\Phi$.

Proposition 6.4. Suppose that the operator $\Phi$ is confluent with respect to $\left(u, x_{0}, \mathbb{S}\right)$ for all $\left(u, x_{0}\right) \in \Omega \times \Xi$. Let $u_{0} \in \mathbb{R}^{n}$. Define $\mathbf{u}_{0} \in \Omega$ by $\mathbf{u}_{0}(t)=u_{0}, \forall t \in \mathbb{R}_{+}$. Then $\forall t \in \mathbb{R}_{+}$ we have $\left[\Phi^{\circ}\left(\mathbf{u}_{0}, x_{0}\right)\right](t)=\left[\Phi^{\circ}\left(\mathbf{u}_{0}, x_{0}\right)\right](0)$.

Proof. The result is a direct consequence of Proposition 6.2. 

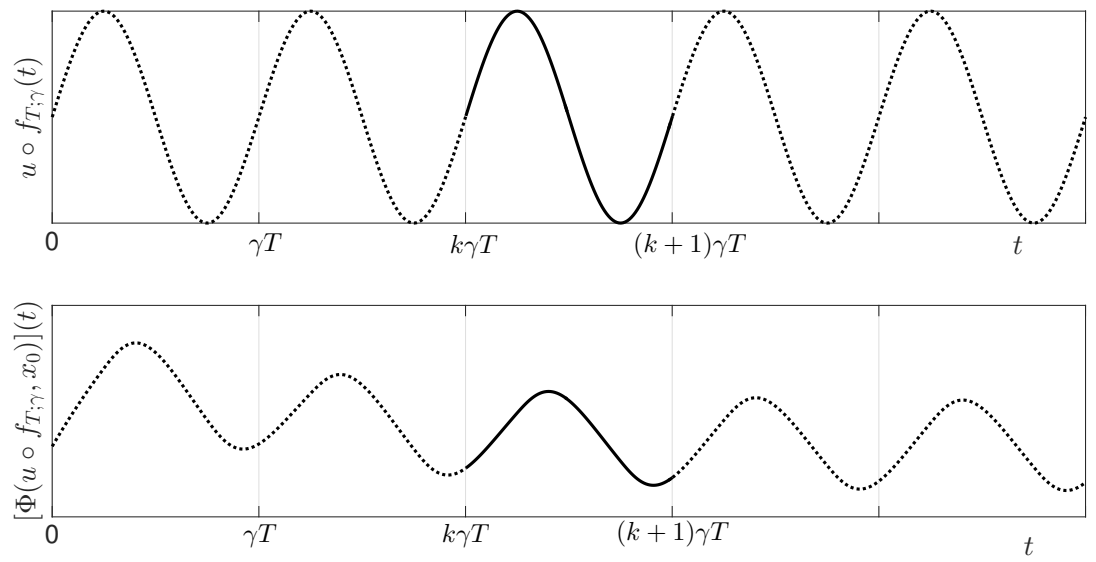

Figure 3: Example of $u \circ f_{T ; \gamma}(t)$ and $\left[\Phi\left(u \circ f_{T ; \gamma}, x_{0}\right)\right](t)$ for $t \in[k \gamma T,(k+1) \gamma T]$ (solid).

\subsection{Inference ( $\mathscr{I} \mathscr{2})$}

We now explore the relationship between confluence and Inference $(\mathscr{I} 2)$.

Let $T \in] 0, \infty\left[, u \in \Omega_{T}\right.$ and $x_{0} \in \Xi$. For any $k \in \mathbb{N}$, any $\left.\gamma \in\right] 0, \infty\left[\right.$, and any $f_{T ; \gamma} \in \mathbb{S}_{T ; \gamma}$ define the set

$$
A_{k ; \gamma}=\left\{\left(u \circ f_{T ; \gamma}(t),\left[\Phi\left(u \circ f_{T ; \gamma}, x_{0}\right)\right](t)\right), t \in[k \gamma T,(k+1) \gamma T]\right\} .
$$

Observe that $u \circ f_{T ; \gamma}$ is $\gamma T$-periodic (see Figure 3). Figure 4 provides the set $A_{k ; \gamma}$ that corresponds to Figure 3 .

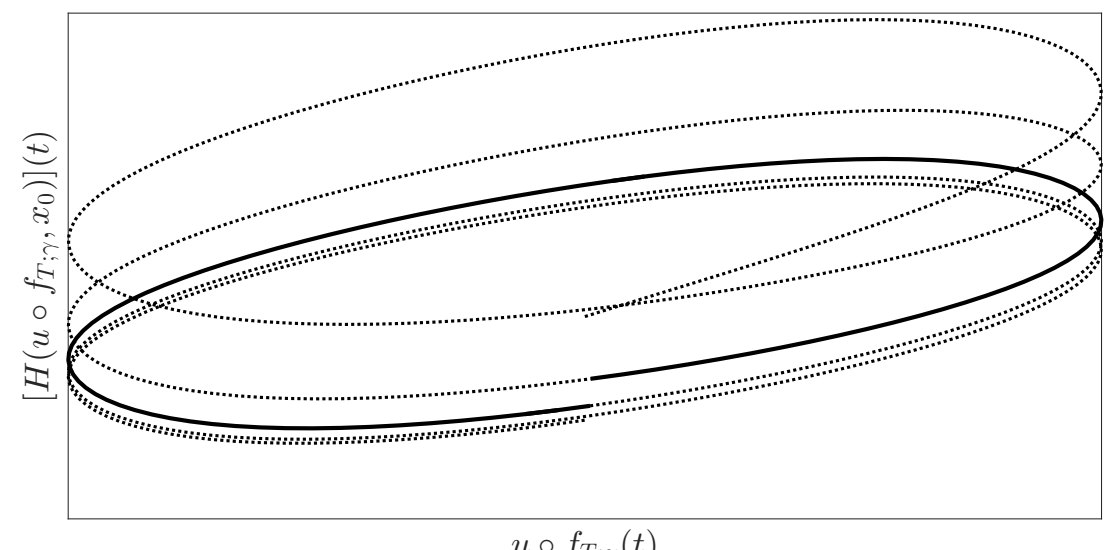

Figure 4: The set $A_{k ; \gamma}$ (solid) that corresponds to Figure 3. 
Note that $A_{k ; \gamma}$ and $\mathcal{Q}_{u ; \Phi ; x_{0}}$ are compact subsets of $\mathbb{R}^{n} \times \Xi$ which is a Banach space with respect to the norm $|(a, b)|=\max (|a|,|b|)$. The Hausdorff distance $h$ between these two sets is

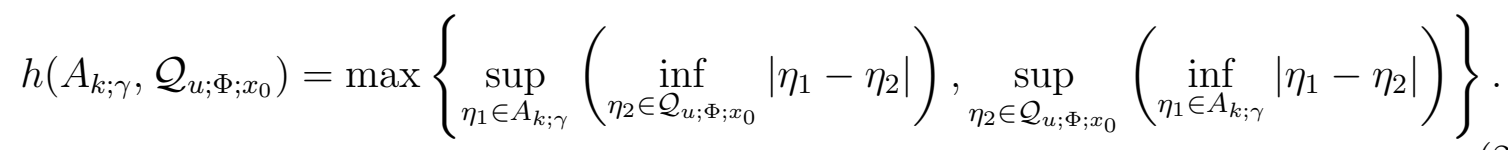

Proposition 6.5. If the operator $\Phi$ is confluent with respect to $\left(u, x_{0}, \mathbb{S}\right)$ then

$$
\lim _{(k, \gamma) \rightarrow \infty}\left(\sup _{f_{T ; \gamma} \in \mathbb{S}_{T ; \gamma}} h\left(A_{k ; \gamma}, \mathcal{Q}_{u ; \Phi ; x_{0}}\right)\right)=0 .
$$

Proof. Observe that

$$
A_{k ; \gamma}=\left\{\left(u(t), \Phi\left(u \circ f_{T ; \gamma}, x_{0}\right) \circ f_{T ; \gamma}^{-1} \circ \tau_{k T}(t)\right), t \in[0, T]\right\} .
$$

In Equation (27) we have for any $\eta_{1} \in A_{k ; \gamma}$

$$
\begin{aligned}
\inf _{\eta_{2} \in \mathcal{Q}_{u ; \Phi ; x_{0}}\left|\eta_{1}-\eta_{2}\right|} & =\inf _{t_{2} \in[0, T]}\left|\left(u\left(t_{1}\right), \Phi\left(u \circ f_{T ; \gamma}, x_{0}\right) \circ f_{T ; \gamma}^{-1} \circ \tau_{k T}\left(t_{1}\right)\right)-\left(u\left(t_{2}\right), \alpha_{u ; \Phi ; x_{0}}\left(t_{2}\right)\right)\right| \\
& \leq\left|\left(u\left(t_{1}\right), \Phi\left(u \circ f_{T ; \gamma}, x_{0}\right) \circ f_{T ; \gamma}^{-1} \circ \tau_{k T}\left(t_{1}\right)\right)-\left(u\left(t_{1}\right), \alpha_{u ; \Phi ; x_{0}}\left(t_{1}\right)\right)\right| \\
& =\left|\Phi\left(u \circ f_{T ; \gamma}, x_{0}\right) \circ f_{T ; \gamma}^{-1} \circ \tau_{k T}\left(t_{1}\right)-\alpha_{u ; \Phi ; x_{0}}\left(t_{1}\right)\right|
\end{aligned}
$$

for some $t_{1} \in[0, T]$, so that

$$
\begin{aligned}
\sup _{\eta_{1} \in A_{k ; \gamma}}\left(\inf _{\eta_{2} \in \mathcal{Q}_{u ; \Phi ; x_{0}}}\left|\eta_{1}-\eta_{2}\right|\right) & \leq \sup _{t_{1} \in[0, T]}\left|\Phi\left(u \circ f_{T ; \gamma}, x_{0}\right) \circ f_{T ; \gamma}^{-1} \circ \tau_{k T}\left(t_{1}\right)-\alpha_{u ; \Phi ; x_{0}}\left(t_{1}\right)\right| \\
& =\left\|\left.\left(\Phi\left(u \circ f_{T ; \gamma}, x_{0}\right) \circ f_{T ; \gamma}^{-1} \circ \tau_{k T}\right)\right|_{[0, T]}-\alpha_{u ; \Phi ; x_{0}}\right\| .
\end{aligned}
$$

For the term $\sup _{\eta_{2} \in \mathcal{Q}_{u ; \Phi ; x_{0}}}\left(\inf _{\eta_{1} \in A_{k ; \gamma}}\left|\eta_{1}-\eta_{2}\right|\right)$, a similar inequality is obtained. Thus

$$
h\left(A_{k ; \gamma}, \mathcal{Q}_{u, \Phi, x_{0}}\right) \leq\left\|\left.\left(\Phi\left(u \circ f_{T ; \gamma}, x_{0}\right) \circ f_{T ; \gamma}^{-1} \circ \tau_{k T}\right)\right|_{[0, T]}-\alpha_{u ; \Phi ; x_{0}}\right\|,
$$

which ends the proof by using Equation (5).

When the operator $\Phi$ is confluent with respect to $\left(u, x_{0}, \mathbb{S}\right)$, Proposition 6.5 says the following: when the input $u \circ f_{T ; \gamma}$ varies slowly -which happens for large $\gamma$ - the graph 
output-versus-input $\left\{\left(u \circ f_{T ; \gamma}, \Phi\left(u \circ f_{T ; \gamma}, x_{0}\right)\right\}\right.$ becomes closer and closer in steady state -that is for $t \geq k \cdot \gamma T$ with a large $k$ - to the hysteresis loop $\mathcal{Q}_{u ; \Phi ; x_{0}}$, see Figure 5 . This is precisely Inference $(\mathscr{I} 2)$.

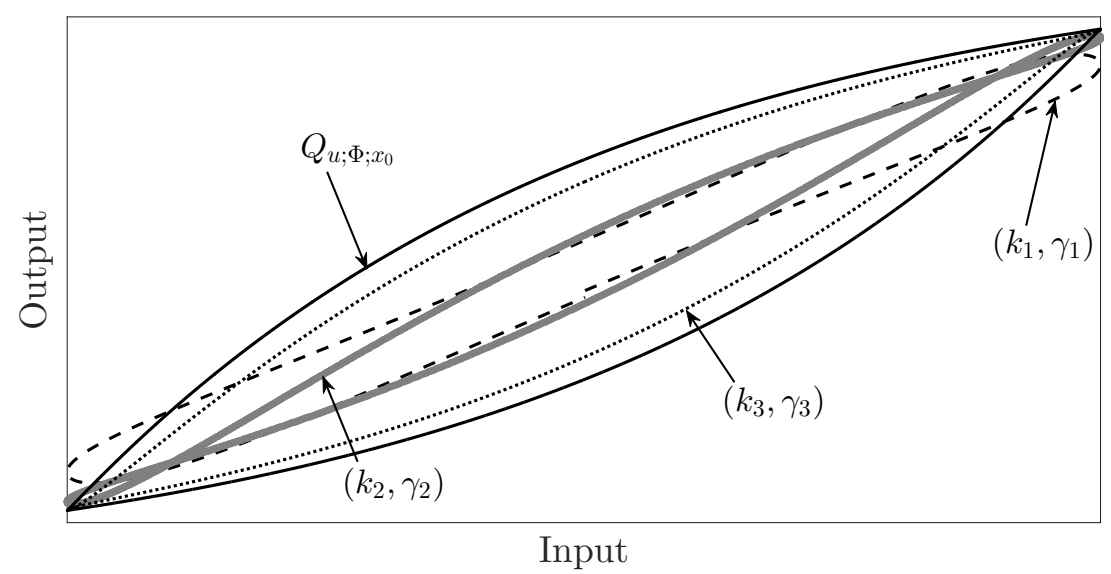

Figure 5: Convergence to $\mathcal{Q}_{u ; \Phi ; x_{0}}$ of the sets $A_{k_{i} ; \gamma_{i}}$ indicated by the arrows $\left(k_{i}, \gamma_{i}\right)$ with $k_{3}>k_{2}>k_{1}$ and $\gamma_{3}>\gamma_{2}>\gamma_{1}$.

\subsection{Inference ( $\mathscr{I} 3)$}

Equation (6) shows that the parametrization of the hysteresis loop comes from the convergence process of Definition 5.1. This is Inference $(\mathscr{I} 3)$.

\section{Modeling rate-dependent hysteresis}

In this section we propose a way to construct some models of rate-dependent hysteresis given a model of a rate-independent one. The flowchart of the model that we propose for the description of the rate-dependent hysteresis is given in Figure 6, which reads

$$
\begin{aligned}
w_{1} & =\mathcal{H}_{1}\left(u, \xi_{1}\right), \\
w_{2} & =\mathcal{H}_{2}\left(u, \xi_{2}\right), \\
v & =w_{1}+w_{2}, \\
z & =\mathcal{L}\left(v, \xi_{3}\right), \\
y & =f \circ z, \\
y & =\mathcal{H}_{3}\left(v, \xi_{3}\right), \\
y & =\mathcal{H}\left(u, x_{0}\right) \text { where } x_{0}=\left(\xi_{1}, \xi_{2}, \xi_{3}\right) .
\end{aligned}
$$




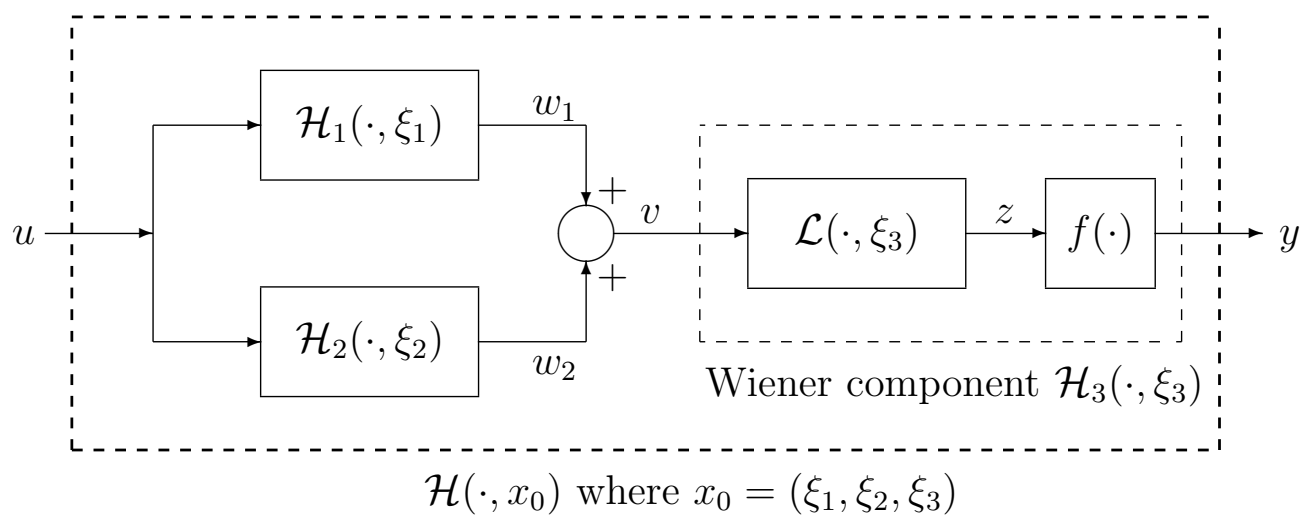

Figure 6: Flowchart of the proposed model.

We describe next the different components of the model.

7.1. The rate-independent component $\mathcal{H}_{1}$

Note that if $f_{1 ; 1} \in \mathbb{I}_{1 ; 1}$ then $f_{1 ; 1}^{-1} \in \mathbb{I}_{1 ; 1}$. Define the set

$$
\mathbb{S}=\left\{f_{1 ; 1} \in \mathbb{I}_{1 ; 1} \mid f_{1 ; 1}, f_{1 ; 1}^{-1} \in W^{1 ; \infty}\left(\mathbb{R}_{+}, \mathbb{R}_{+}\right)\right\} .
$$

For any $d \in[1, \infty[$ and any $T, \gamma \in] 0, \infty[$ define the set

$\mathbb{S}_{T ; \gamma ; d}^{*}=\left\{f_{T ; \gamma}=f_{T ; 1} \circ s_{\gamma}\right.$ where $f_{T ; 1}=s_{\frac{1}{T}} \circ f_{1 ; 1} \circ s_{T} \mid f_{1 ; 1} \in \mathbb{S},\left\|\dot{f}_{1 ; 1}\right\| \leq d$ and $\left.\|\overbrace{1 ; 1}^{-1}\| \leq d\right\}$.

Note that Id $\in \mathbb{S}_{1 ; 1 ; d}^{*},\left\|\dot{f}_{T ; 1}\right\| \leq d$, and $\left\|\stackrel{\dot{f_{T ; 1}^{-1}}}{ }\right\| \leq d$

Define also the set

$$
\mathbb{S}_{T ; d}^{*}=\bigcup_{\gamma \in] 0, \infty[} \mathbb{S}_{T ; \gamma ; d}^{*}
$$

Let $\Xi_{1}$ be a set of initial conditions and $\mathscr{U} \subset C^{0}\left(\mathbb{R}_{+}, \mathbb{R}^{n}\right)$ be such that $\left.\forall T \in\right] 0, \infty[$, $\forall u \in \mathscr{U} \cap \Omega_{T}$, we have $u \circ \mathbb{S}_{T ; d}^{*} \subset \mathscr{U}$. We assume that the operator $\mathcal{H}_{1}: \mathscr{U} \times \Xi_{1} \rightarrow$ $W^{1 ; \infty}\left(\mathbb{R}_{+}, \mathbb{R}^{m}\right)$ satisfies (i)-(ii).

(i) $\mathcal{H}_{1}$ is $\left(\{u\}, \mathbb{S}_{T ; d}^{*}, \Xi_{1}\right)$-rate independent for any $\left.(T, u) \in\right] 0, \infty\left[\times \mathscr{U} \cap \Omega_{T}\right.$.

(ii) $\mathcal{H}_{1}$ is confluent with respect to $\left(u, \xi_{1}, \mathbb{S}_{1 ; 1 ; d}^{*}\right)$ for any $\left(u, \xi_{1}\right) \in \mathscr{U} \cap \Omega \times \Xi_{1}$. 


\subsection{The component $\mathcal{H}_{2}$}

Let $\Xi_{2}$ be a set of initial conditions. We assume that the operator $\mathcal{H}_{2}: \mathscr{U} \times \Xi_{2} \rightarrow$ $L^{\infty}\left(\mathbb{R}_{+}, \mathbb{R}^{m}\right)$ satisfies (i)-(ii).

(i) $\left.\forall\left(T, u, \xi_{2}\right) \in\right] 0, \infty\left[\times \mathscr{U} \cap \Omega_{T} \times \Xi_{2}, \exists c_{u ; \xi_{2}} \in \mathbb{R}_{+}, \exists \gamma_{u ; \xi_{2}} \in\right] 0, \infty[$ such that $\forall \gamma \in$ $\left[\gamma_{u ; \xi_{2}}, \infty[\right.$ we have

$$
\sup _{f_{T ; \gamma} \in \mathbb{S}_{T ; \gamma ; d}^{*}}\left\|\mathcal{H}_{2}\left(u \circ f_{T ; \gamma}, \xi_{2}\right)\right\| \leq c_{u ; \xi_{2}}
$$

(ii) For any $T \in] 0, \infty\left[\right.$ define the function $\left.h: \mathscr{U} \cap \Omega_{T} \times \Xi_{2} \times \mathbb{N} \times\right] 0, \infty\left[\rightarrow \mathbb{R}_{+} \cup\{\infty\}\right.$ by

$$
h\left(u, \xi_{2}, k, \gamma\right)=\sup _{f_{T ; \gamma} \in \mathbb{S}_{T ; \gamma ; d}^{*}}\left\|\left.\mathcal{H}_{2}\left(u \circ f_{T ; \gamma}, \xi_{2}\right)\right|_{[k \gamma T, \infty[\|}\right\|
$$

Then, $\quad \lim _{(k, \gamma) \rightarrow \infty} h\left(u, \xi_{2}, k, \gamma\right)=0, \forall\left(u, \xi_{2}\right) \in \mathscr{U} \cap \Omega_{T} \times \Xi_{2}$.

Proposition 7.1. Let $u_{0} \in \mathbb{R}^{n}$. Define $\mathbf{u}_{0}: \mathbb{R}_{+} \rightarrow \mathbb{R}^{n}$ by $\mathbf{u}_{0}(t)=u_{0}, \forall t \in \mathbb{R}_{+}$. If $\mathbf{u}_{0} \in \mathscr{U}$ then $\forall \xi_{2} \in \Xi_{2}$ we have $\lim _{t \rightarrow \infty}\left[\mathcal{H}_{2}\left(\mathbf{u}_{0}, \xi_{2}\right)\right](t)=0$.

Proof. Immediate from (40).

Owing to Proposition 7.1 we call $\mathcal{H}_{2}$ the capacitor since in electrical circuits a constant voltage gives rise to a decaying current in a capacitor.

\subsection{The Wiener component $\mathcal{H}_{3}$}

The Wiener component is given by the composition of a -possibly nonlinear- function $f$ and a linear system $\mathcal{L}$ as follows (see for instance Ref. [19]):

$$
\begin{aligned}
\dot{x}(t) & =A x(t)+B v(t), \\
z(t) & =C x(t), \\
x(0) & =\xi_{3} \in \mathbb{R}^{p}, \\
y(t) & =f(z(t)),
\end{aligned}
$$

where $t \in \mathbb{R}_{+}, A \in \mathbb{R}^{p \times p}$ is stable, $\dot{x}=\frac{\mathrm{d} x}{\mathrm{~d} t}, B \in \mathbb{R}^{p \times m}, C \in \mathbb{R}^{r \times p}$ where $r, p \in \mathbb{N} \backslash\{0\}$. Observe that $v \in L^{\infty}\left(\mathbb{R}_{+}, \mathbb{R}^{m}\right)$ so that $x \in W^{1, \infty}\left(\mathbb{R}_{+}, \mathbb{R}^{p}\right)$ and $z \in W^{1, \infty}\left(\mathbb{R}_{+}, \mathbb{R}^{r}\right)$. The function $f: \mathbb{R}^{r} \rightarrow \mathbb{R}^{q}$ where $q \in \mathbb{N} \backslash\{0\}$ is assumed to be continuous so that $y \in$ $L^{\infty}\left(\mathbb{R}_{+}, \mathbb{R}^{q}\right) \cap C^{0}\left(\mathbb{R}_{+}, \mathbb{R}^{q}\right)$. 
We have $\mathcal{L}: L^{\infty}\left(\mathbb{R}_{+}, \mathbb{R}^{m}\right) \times \mathbb{R}^{p} \rightarrow L^{\infty}\left(\mathbb{R}_{+}, \mathbb{R}^{r}\right) \cap C^{0}\left(\mathbb{R}_{+}, \mathbb{R}^{r}\right)$ and $\mathcal{H}_{3}: L^{\infty}\left(\mathbb{R}_{+}, \mathbb{R}^{m}\right) \times$ $\mathbb{R}^{p} \rightarrow L^{\infty}\left(\mathbb{R}_{+}, \mathbb{R}^{q}\right) \cap C^{0}\left(\mathbb{R}_{+}, \mathbb{R}^{q}\right)$.

Equation (41) is satisfied for almost all $t \in \mathbb{R}_{+}$whilst Equations (42) and (44) are satisfied for all $t \in \mathbb{R}_{+}$.

Note that since the matrix $A$ is stable, it is invertible. The static gain of the linear subsystem (41)-(42) is given by the matrix $G=-C A^{-1} B,[2$, p. 60].

\subsection{The operator $\mathcal{H}$}

Denote $\Xi=\Xi_{1} \times \Xi_{2} \times \mathbb{R}^{p}$. Then $\mathcal{H}: \mathscr{U} \times \Xi \rightarrow L^{\infty}\left(\mathbb{R}_{+}, \mathbb{R}^{q}\right) \cap C^{0}\left(\mathbb{R}_{+}, \mathbb{R}^{q}\right)$ with $y=\mathcal{H}\left(u, x_{0}\right)$.

\subsection{Confluence of the operator $\mathcal{H}$}

Theorem 7.1 is the main result of Section 7. This theorem says that the hysteresis loop of the overall model $\mathcal{H}$ can be calculated from the hysteresis loop of its rate-independent component $\mathcal{H}_{1}$.

An example of the application of this theorem is provided in Section 7.6 where the rate-independent component $\mathcal{H}_{1}$ is the Dahl model.

Theorem 7.1. $\forall\left(u, x_{0}\right) \in \mathscr{U} \cap \Omega \times \Xi$ we have

(i) $\mathcal{H}$ is confluent with respect to $\left(u, x_{0}, \mathbb{S}_{1 ; 1 ; d}^{*}\right)$.

(ii) $\alpha_{u ; \mathcal{H} ; x_{0}}=f \circ\left(G \alpha_{u ; \mathcal{H}_{1} ; \xi_{1}}\right)$.

Proof. See Appendix B.

Observe that Theorem 7.1 (ii) relates the canonical decompositions of $\mathcal{H}$ and $\mathcal{H}_{1}$ as $\mathcal{H}^{\circ}=f \circ\left(G \mathcal{H}_{1}^{\circ}\right)$.

\subsection{Numerical example}

Our aim in this section is to illustrate the concepts introduced in this paper using a numerical example.

Consider the rate-independent component $\mathcal{H}_{1}\left(u, \xi_{1}\right)=w_{1}$ given by the Dahl model for dry friction [15, Equations (10)-(11)]:

$$
\begin{aligned}
\dot{w}_{1}(t) & =\dot{u}(t)-|\dot{u}(t)| w_{1}(t), \text { for almost all } t \in \mathbb{R}_{+}, \\
w_{1}(0) & =\xi_{1},
\end{aligned}
$$

where $u \in W^{1 ; \infty}\left(\mathbb{R}_{+}, \mathbb{R}\right), \xi_{1} \in[-1,1]$, and $n=m=1$. Then $w_{1} \in W^{1 ; \infty}\left(\mathbb{R}_{+}, \mathbb{R}\right)$ (see the proof of $\left[17\right.$, Theorem 1]) so that $\mathcal{H}_{1}: W^{1 ; \infty}\left(\mathbb{R}_{+}, \mathbb{R}\right) \times[-1,1] \rightarrow W^{1 ; \infty}\left(\mathbb{R}_{+}, \mathbb{R}\right)$. Take $d=10$ ( $d$ is defined at the beginning of Section 7.1). 
Proposition 7.2. $\mathcal{H}_{1}$ is $\left(W^{1 ; \infty}\left(\mathbb{R}_{+}, \mathbb{R}\right), \mathbb{S}_{1 ; 1 ; d}^{*},[-1,1]\right)$-rate independent.

Proof. Let $g \in \mathbb{S}_{1 ; 1 ; d}^{*}$. Define $w_{g} \in W^{1 ; \infty}\left(\mathbb{R}_{+}, \mathbb{R}\right)$ as the solution of

$$
\begin{aligned}
\dot{w}_{g}(t) & =\overparen{u \circ g}(t)-|\stackrel{\cdot}{u \circ g}(t)| w_{g}(t), \text { for almost all } t \in \mathbb{R}_{+}, \\
w_{g}(0) & =\xi_{1} .
\end{aligned}
$$

Define $\tau=g(t)$. Then, using Proposition Appendix A.1 and the fact that $g$ maps zeromeasure sets to zero-measure sets, Equations (47)-(48) give

$$
\begin{aligned}
& \dot{w}_{g}\left(g^{-1}(\tau)\right)=\dot{g}\left(g^{-1}(\tau)\right)\left(\dot{u}(\tau)-|\dot{u}(\tau)| w_{g}\left(g^{-1}(\tau)\right)\right), \text { for almost all } \tau \in \mathbb{R}_{+}, \\
& w_{g}\left(g^{-1}(0)\right)=\xi_{1}
\end{aligned}
$$

Since $\|\overbrace{g^{-1}}\| \leq d$ it comes that $\dot{g}\left(g^{-1}(\tau)\right)$ can be zero or undefined only on a set of measure zero. Thus (49)-(50) give

$\overparen{\left(w_{g} \circ g^{-1}\right)}(\tau)=\frac{1}{\dot{g}\left(g^{-1}(\tau)\right)} \dot{w}_{g}\left(g^{-1}(\tau)\right)=\dot{u}(\tau)-|\dot{u}(\tau)|\left(w_{g} \circ g^{-1}\right)(\tau)$, for almost all $\tau \in \mathbb{R}_{+}$,

$\left(w_{g} \circ g^{-1}\right)(0)=\xi_{1}$.

Comparing (45)-(46) and (51)-(52), and taking into account that (45)-(46) has a unique solution, it comes that $w_{g} \circ g^{-1}=w_{1}$. Thus, $\mathcal{H}_{1}\left(u \circ g, \xi_{1}\right)=w_{g}=w_{1} \circ g=\mathcal{H}_{1}\left(u, \xi_{1}\right) \circ g$.

Take $u$ to be the $2-$ periodic input of Figure 7 such that

$$
u(t)= \begin{cases}t, & t \in[0,1], \\ 2-t, & t \in[1,2]\end{cases}
$$

Then, [20, Theorem 3, p. 47] shows that Equality (B.8) holds with

$$
\alpha_{u ; \mathcal{H}_{1} ; \xi_{1}}(t)= \begin{cases}1-\frac{2}{1+e^{-1}} e^{-t}, & t \in[0,1], \\ -1+\frac{2}{1+e^{-1}} e^{1-t}, & t \in[1,2],\end{cases}
$$

see also Ref. [15, Equations (30) and (33)-(34)]. Thus $\mathcal{H}_{1}$ satisfies Conditions (i) and (ii) of Section 7.1. 


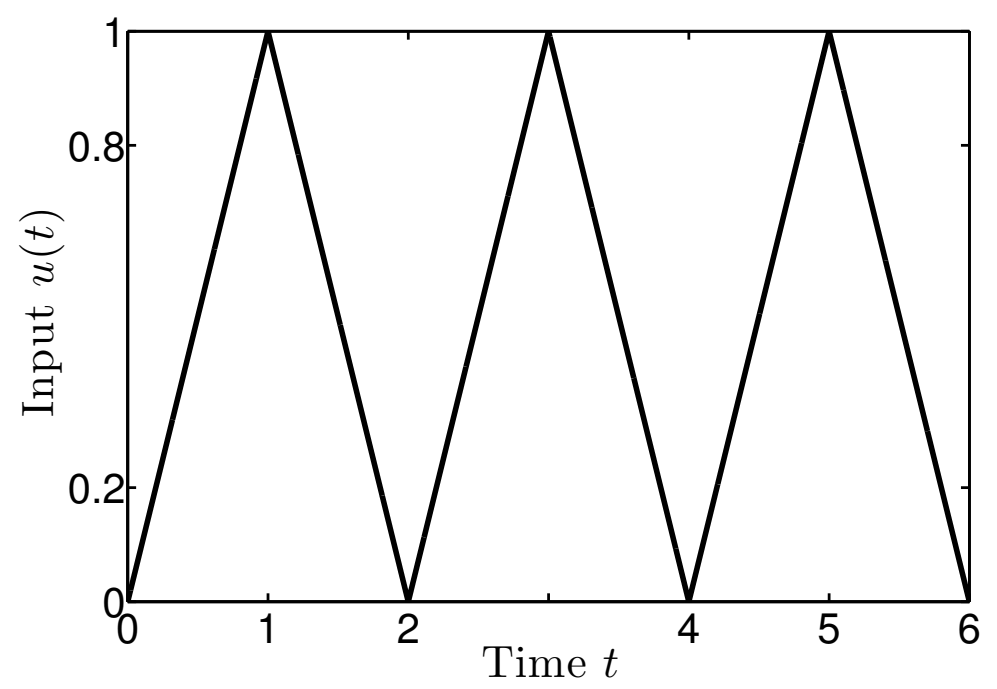

Figure 7: $u(t)$ versus $t$.

Equation (53) gives the explicit expression of the hysteresis loop $\mathcal{Q}_{u ; \mathcal{H}_{1} ; \xi_{1}}$ defined by Equation (9). This loop is plotted in Figure 8.

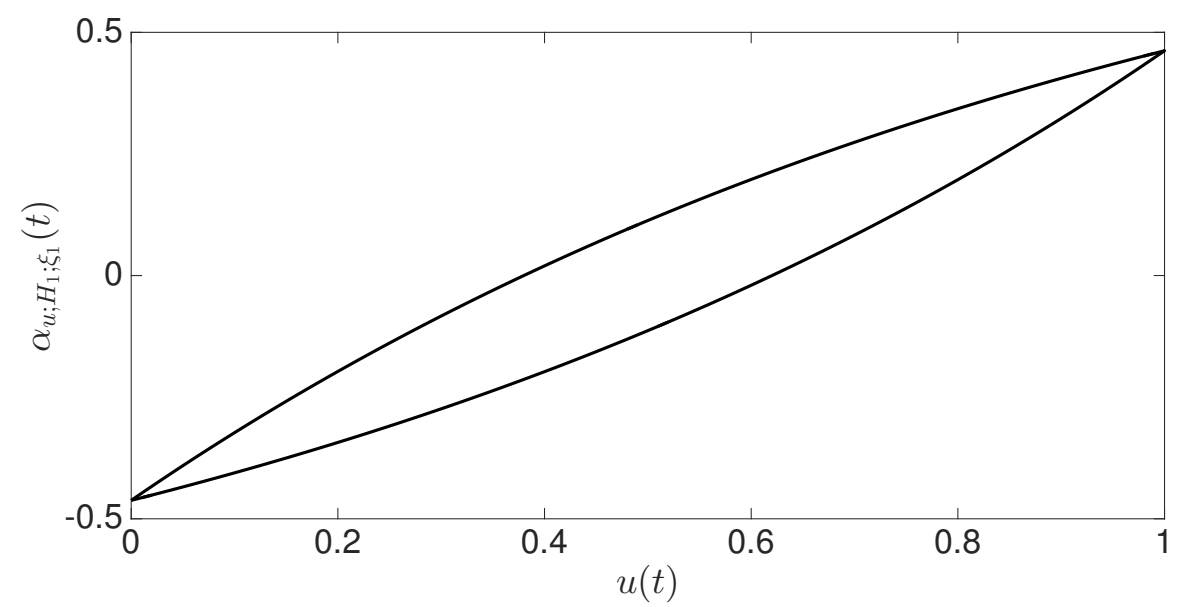

Figure 8: Hysteresis loop $\mathcal{Q}_{u ; \mathcal{H}_{1} ; \xi_{1}}$. 


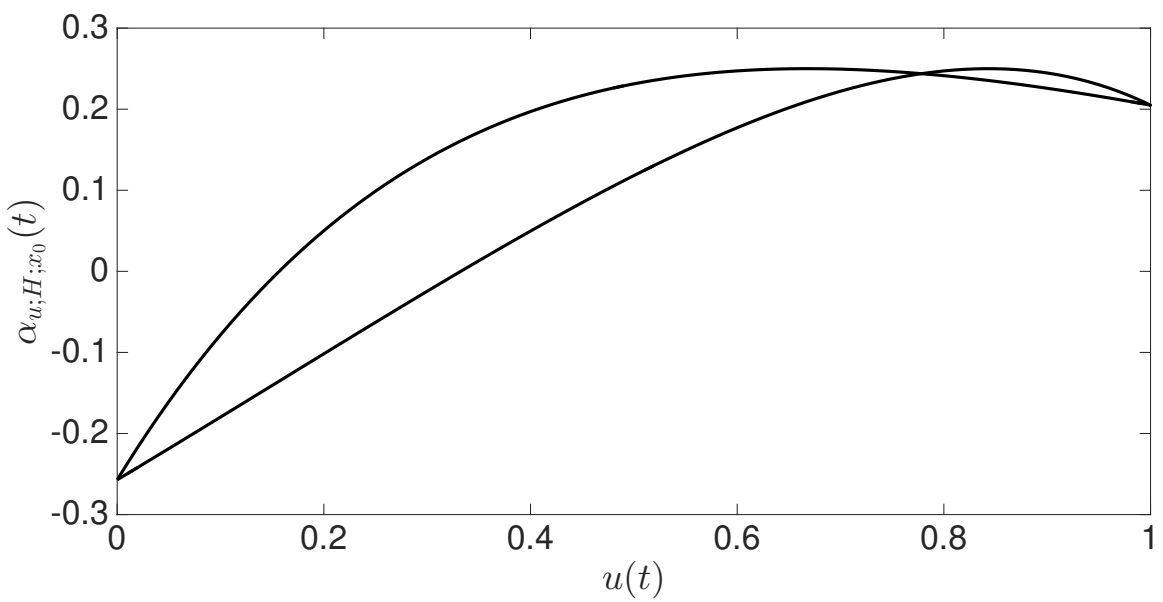

Figure 9: Hysteresis loop $\mathcal{Q}_{u ; \mathcal{H} ; x_{0}}$.

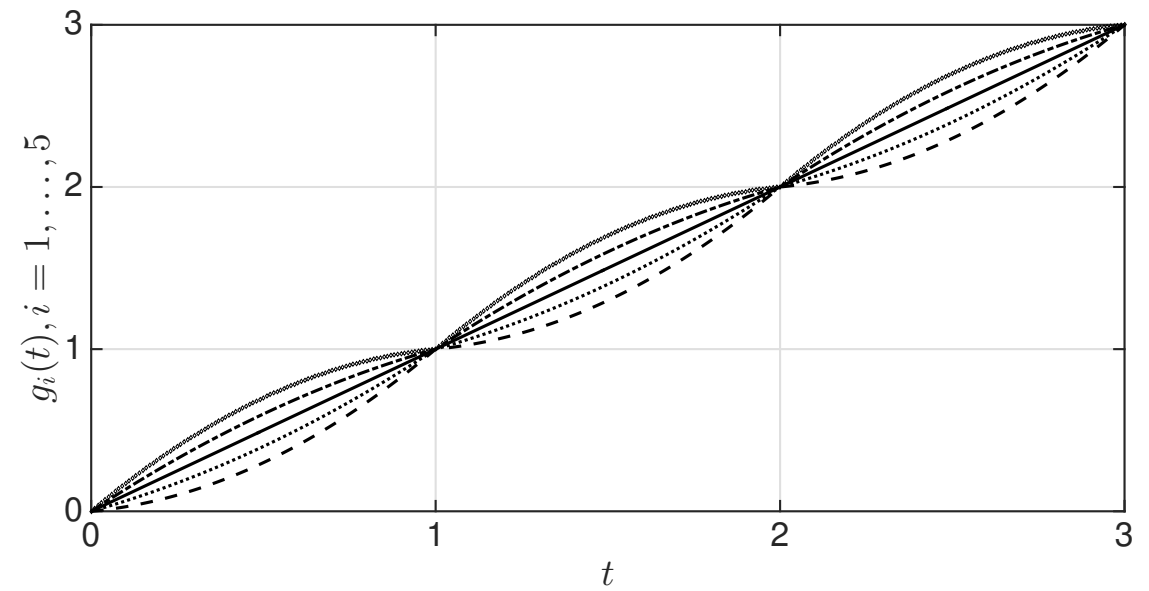

Figure 10: Functions $g_{i}, i=1, \ldots, 5$. 


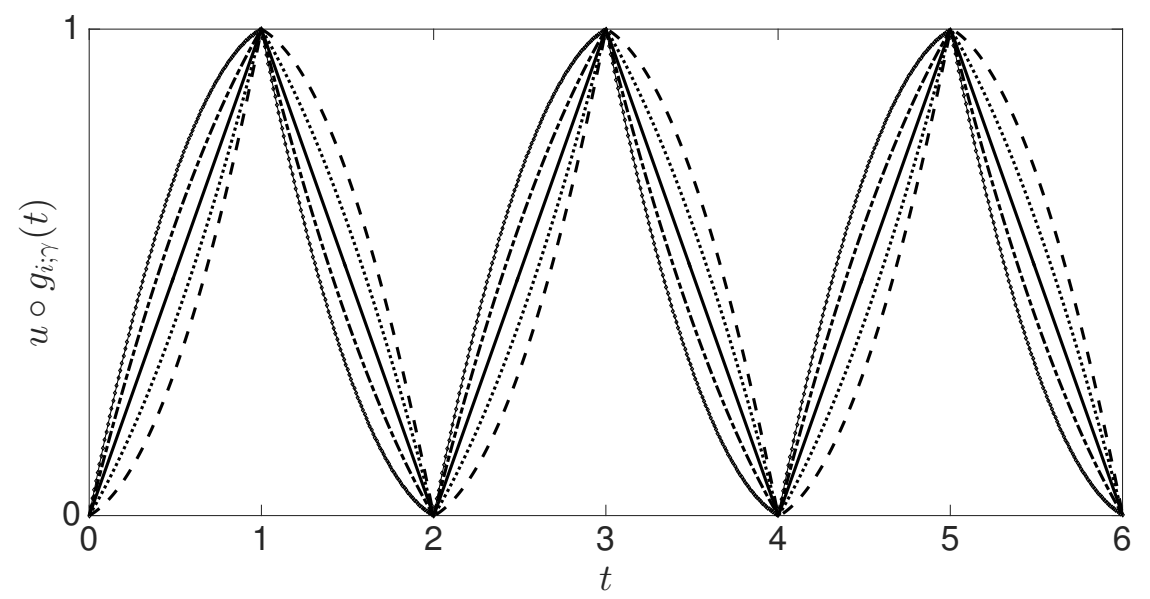

Figure 11: Inputs $u \circ g_{i ; \gamma}, i=1, \ldots, 5$ for $\gamma=1$.

Consider the capacitor $\mathcal{H}_{2}(u)=\dot{u}$. In this case $\Xi_{2}=\emptyset$ and $\mathcal{H}_{2}: W^{1 ; \infty}\left(\mathbb{R}_{+}, \mathbb{R}\right) \rightarrow$ $L^{\infty}\left(\mathbb{R}_{+}, \mathbb{R}\right)$. Using Proposition Appendix A.1 it can be checked that $\mathcal{H}_{2}$ satisfies Condition (i) of Section 7.2 with $\gamma_{u}=1$ and $c_{u}=d\|\dot{u}\|=10$. Condition (ii) of Section 7.2 is also readily checked.

The linear subsystem is given by $A=-1, B=1, C=1, r=p=1$, that is

$$
\begin{aligned}
\dot{x}(t) & =-x(t)+v(t), \text { for almost all } t \in \mathbb{R}_{+}, \\
x(0) & =\xi_{3} \in \mathbb{R}, \\
z(t) & =x(t) .
\end{aligned}
$$

In this case the static gain is $G=1$.

Finally, the function $f: \mathbb{R} \rightarrow \mathbb{R}$ is given by

$$
f(\zeta)=\frac{1}{4}-\left(\zeta-\frac{1}{4}\right)^{2}, \forall \zeta \in \mathbb{R} .
$$

Define $\Xi=[-1,1] \times \mathbb{R}$.

Theorem 7.1 shows that the operator $\mathcal{H}: W^{1 ; \infty}\left(\mathbb{R}_{+}, \mathbb{R}\right) \times \Xi \rightarrow L^{\infty}\left(\mathbb{R}_{+}, \mathbb{R}\right) \cap C^{0}\left(\mathbb{R}_{+}, \mathbb{R}\right)$ of Figure 6 is confluent with respect to $\left(u, x_{0}, \mathbb{S}_{1 ; 1 ; 10}^{*}\right)$, where $x_{0}=\left(\xi_{1}, \xi_{3}\right)$, and that $\alpha_{u ; \mathcal{H} ; x_{0}}=f \circ \alpha_{u ; \mathcal{H}_{1} ; \xi_{1}}$. Using Equation (53) we obtain

$$
\alpha_{u ; \mathcal{H} ; x_{0}}(t)= \begin{cases}\frac{1}{4}-\left(\frac{3}{4}-\frac{2}{1+e^{-1}} e^{-t}\right)^{2}, & t \in[0,1], \\ \frac{1}{4}-\left(-\frac{5}{4}+\frac{2}{1+e^{-1}} e^{1-t}\right)^{2}, & t \in[1,2] .\end{cases}
$$




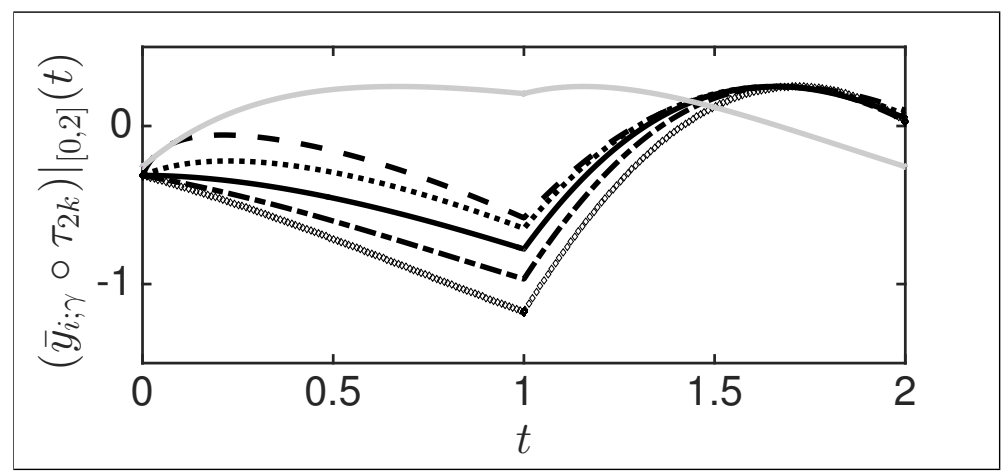

(a) $\gamma=1, k=0$.

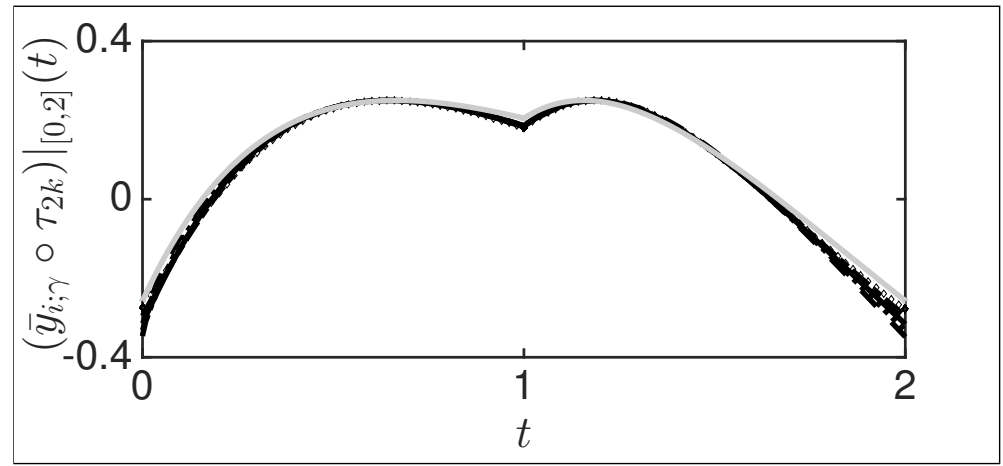

(b) $\gamma=10, k=3$.

Figure 12: Black, $\left.\left(\bar{y}_{i ; \gamma} \circ \tau_{2 k}\right)\right|_{[0,2]}(t), i=1, \ldots, 5$ versus $t$. Grey, $\alpha_{u ; \mathcal{H} ; x_{0}}(t)$ versus $t$.

Equation (57) provides the explicit expression of the hysteresis loop $\mathcal{Q}_{u ; \mathcal{H} ; x_{0}}$. This loop is plotted in Figure 9.

If the function $f$ were the identity function we would have had $\mathcal{Q}_{u ; \mathcal{H} ; x_{0}}=\mathcal{Q}_{u ; \mathcal{H}_{1} ; \xi_{1}}$. The role of a nonlinear function $f$ is to get a hysteresis loop $\mathcal{Q}_{u ; \mathcal{H} ; x_{0}}$ that does not have the same shape as $\mathcal{Q}_{u ; \mathcal{H}_{1} ; \xi_{1}}$.

To illustrate graphically Equation (8) we need to be able to compute numerically the quantity

$$
\wp_{\mathcal{H}}(k, \gamma)=\sup _{f_{2 ; \gamma} \in \mathbb{S}_{2 ; \gamma ; 10}^{*}}\left\|\left.\left(\mathcal{H}\left(u \circ f_{2 ; \gamma}, x_{0}\right) \circ f_{2 ; \gamma}^{-1} \circ \tau_{2 k}\right)\right|_{[0,2]}-\alpha_{u ; \mathcal{H} ; x_{0}}\right\|
$$

for any given $(k, \gamma) \in \mathbb{N} \times] 0, \infty\left[\right.$. This, however, is not possible because the set $\mathbb{S}_{2 ; \gamma ; 10}^{*}$ has an infinite number of elements. This is why we consider instead the quantity

$$
\wp_{\mathcal{H}}^{\prime}(k, \gamma)=\sup _{f_{2 ; \gamma} \in \mathbb{S}_{\gamma}^{\prime}}\left\|\left.\left(\mathcal{H}\left(u \circ f_{2 ; \gamma}, x_{0}\right) \circ f_{2 ; \gamma}^{-1} \circ \tau_{2 k}\right)\right|_{[0,2]}-\alpha_{u ; \mathcal{H} ; x_{0}}\right\| \leq \wp_{\mathcal{H}}(k, \gamma)
$$




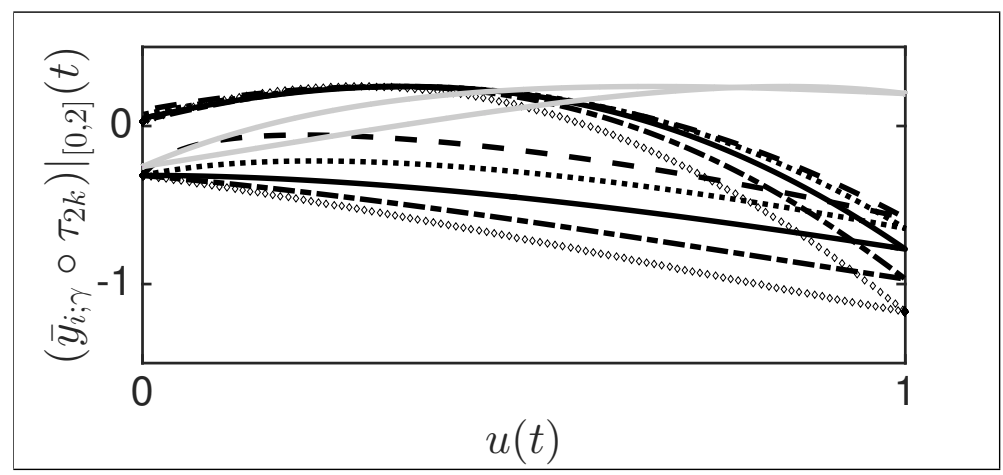

(a) $\gamma=1, k=0$.

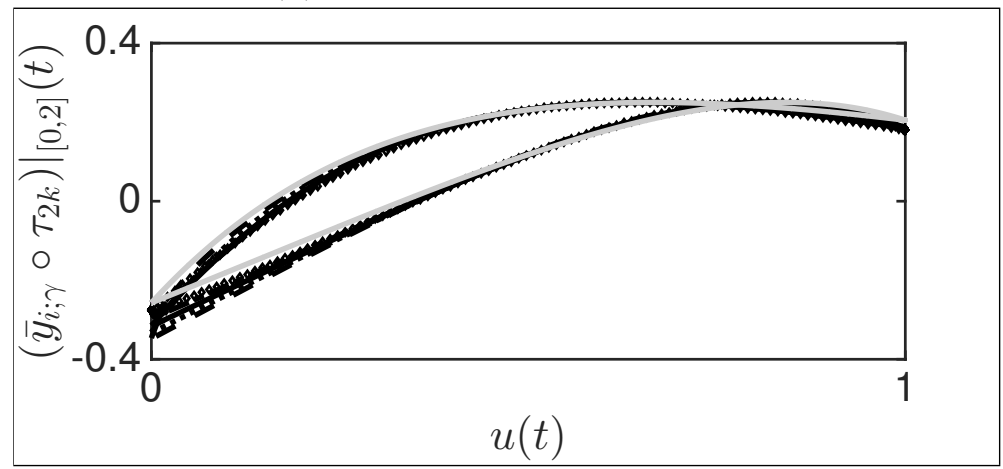

(b) $\gamma=10, k=3$.

Figure 13: Black, Sets $A_{k ; \gamma}, i=1, \ldots, 5$. Grey, Hysteresis loop $\mathcal{Q}_{u ; \mathcal{H} ; x_{0}}$.

where $\mathbb{S}_{\gamma}^{\prime} \ni s_{\gamma}$ is a finite subset of $\mathbb{S}_{2 ; \gamma ; 10}^{*}$. The set $\mathbb{S}_{\gamma}^{\prime}$ is constructed as follows. Consider the functions $g_{i} \in \mathbb{S}_{1 ; 1 ; 10}^{*}, i=1, \ldots, 5$ of Figure 10 with $g_{1}=\mathrm{Id}$. Then

$$
\mathbb{S}_{\gamma}^{\prime}=\left\{g_{i ; \gamma}=s_{\frac{1}{2}} \circ g_{i} \circ s_{2 \gamma}, i=1, \ldots, 5\right\} .
$$

The inputs $u \circ g_{i ; 1}, i=1, \ldots, 5$ are given in Figure 11 .

From Equation (B.21) it comes that the function $\bar{y}_{i ; \gamma}=\mathcal{H}\left(u \circ g_{i ; \gamma}, x_{0}\right) \circ g_{i ; \gamma}^{-1}$ is given by

$$
\bar{y}_{i ; \gamma}(t)=f(\exp \left(-g_{i ; \gamma}^{-1}(t)\right) \xi_{3}+\int_{0}^{t} \exp \left(g_{i ; \gamma}^{-1}(\tau)-g_{i ; \gamma}^{-1}(t)\right) \overbrace{g_{i ; \gamma}^{-1}}^{\dot{2}}(\tau)\left(w_{1}(\tau)+\bar{w}_{2 ; \gamma}(\tau)\right) \mathrm{d} \tau)
$$

where $w_{1}$ is the solution of (45)-(46) and

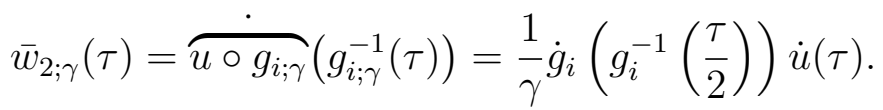


Figure 12 gives the functions $\left.\left(\bar{y}_{i ; \gamma} \circ \tau_{2 k}\right)\right|_{[0,2]}, i=1, \ldots, 5$ and $\alpha_{u ; \mathcal{H} ; x_{0}}$. It can be seen that, as $\gamma$ and $k$ increase, $\left.\left(\bar{y}_{i ; \gamma} \circ \tau_{2 k}\right)\right|_{[0,2]}, i=1, \ldots, 5$ converge to $\alpha_{u ; \mathcal{H} ; x_{0}}$ as predicted by Theorem 7.1.

Figure 13 shows the convergence of the sets $A_{k ; \gamma}, i=1, \ldots, 5$ to the hysteresis loop $\mathcal{Q}_{u ; \mathcal{H} ; x_{0}}$. Figure 14 gives the function $\wp_{\mathcal{H}}^{\prime}$. It can be seen that, as $\gamma$ and $k$ increase, $\wp_{\mathcal{H}}^{\prime}(k, \gamma)$ goes to zero owing to the confluence of the operator $\mathcal{H}$.

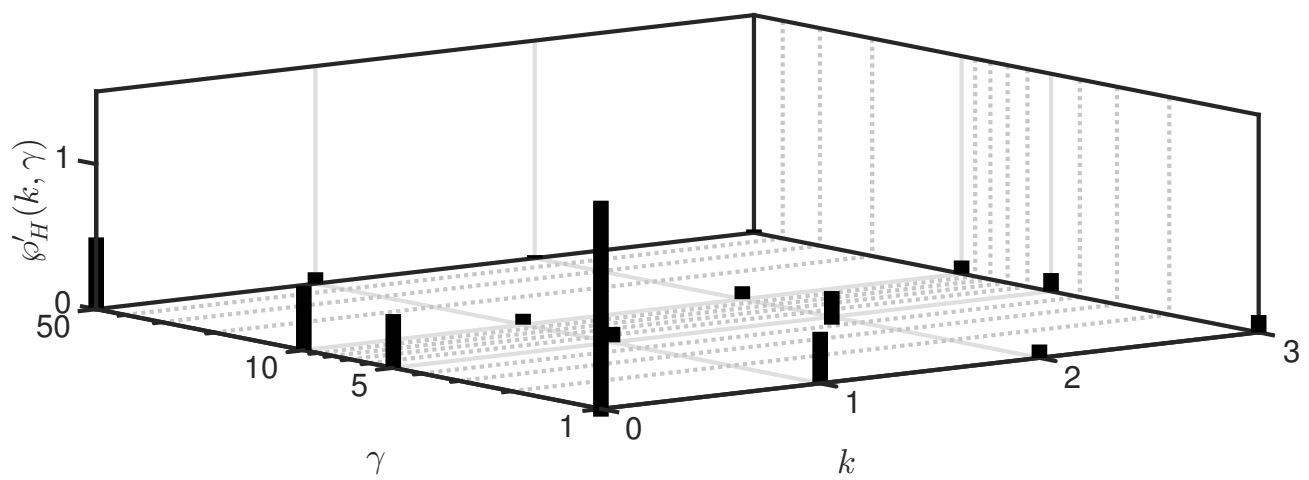

Figure 14: $\wp_{\mathcal{H}}^{\prime}(k, \gamma)$ versus $(k, \gamma)$ for $k=0,1,2,3$ and $\gamma=1,5,10,50$.

\section{Conclusion}

By 'continuous hysteresis' we mean that a continuous input leads to a continuous output and a continuous hysteresis loop. For this class of processes we have achieved the goals we have set: using a general enough frame, and with a single premise we have obtained analytically the set of inferences $\{(\mathscr{I} 1),(\mathscr{I} 2),(\mathscr{I} 3),(\mathscr{I} 4)\}$ through the concept of confluence. We have also applied this concept to elaborate a model that produces rate-dependent hysteresis systems from rate-independent ones.

\section{Appendix A.}

Proposition Appendix A.1. Let $n \in \mathbb{N} \backslash\{0\}, u \in W^{1 ; \infty}\left(\mathbb{R}_{+}, \mathbb{R}^{n}\right)$ and $f$ a homeomorphism of $\mathbb{R}_{+}$such that $f, f^{-1} \in W^{1 ; \infty}\left(\mathbb{R}_{+}, \mathbb{R}\right)$. Then $u \circ f \in W^{1 ; \infty}\left(\mathbb{R}_{+}, \mathbb{R}^{n}\right)$ and $\overparen{u \circ f}(t)=\dot{f}(t) \dot{u}(f(t))$ for almost all $t \in \mathbb{R}_{+}$where $\dot{f}(t) \dot{u}(f(t))$ is interpreted to be zero whenever $\dot{f}(t)=0$ (even if $u$ is not differentiable at $f(t)$ ).

Proof. Take $n=1$, then Ref. [24, Exercise 3.51] shows that $u \circ f$ is locally absolutely continuous whilst Ref. [24, Corollary 3.50] shows that the chain rule holds. The function 
$\dot{u} \circ f$ is measurable since $\dot{u}$ is measurable and $f^{-1}$ maps measurable sets into measurable sets $[24$, p. 83]. To show that $\|\overparen{u \circ f}\|<\infty$ we proceed as follows. Define $A=\{t \in$ $\left.\mathbb{R}_{+}|| \dot{u}(t) \mid>\|\dot{u}\|\right\}$, then $\mu(A)=0$. Since $f^{-1}$ is absolutely continuous it follows that $\mu\left(f^{-1}(A)\right)=0$ with $f^{-1}(A)=\left\{t \in \mathbb{R}_{+} \mid(\dot{u} \circ f)(t)>\|\dot{u}\|\right\}$. This fact shows that $\|\dot{u} \circ f\| \leq$ $\|\dot{u}\|<\infty$. Define $B=\left\{t \in \mathbb{R}_{+}|| \dot{f}(t) \mid>\|\dot{f}\|\right\}, C=\left\{t \in \mathbb{R}_{+}||(\dot{u} \circ f)(t) \mid>\|\dot{u} \circ f\|\right\}$ and

$\left.D=\left\{t \in \mathbb{R}_{+}|| \widehat{(u \circ f}\right)(t) \mid>\|\dot{f}\| \cdot\|\dot{u} \circ f\|\right\}$, then $\mu(B)=\mu(C)=0$ and $D \subset B \cup C \cup E$ where $E$ is the set of measure zero on which the chain rule does not hold. This means that $\mu(D)=0$ which implies that $\|\overparen{u \circ f}\| \leq\|\dot{f}\| \cdot\|\dot{u} \circ f\|<\infty$.

This fact shows that $u \circ f$ is absolutely continuous on $\mathbb{R}_{+}$. The case $n>1$ follows similarly.

\section{Appendix B. Proof of Theorem 7.1}

Proof. Let $\left.\left(T, u, x_{0}\right) \in\right] 0, \infty\left[\times \mathscr{U} \cap \Omega_{T} \times \Xi\right.$. For any $\left.\gamma \in\right] 0, \infty\left[\right.$ let $f_{T ; \gamma} \in \mathbb{S}_{T ; \gamma ; d}^{*}$. Define

$$
\begin{aligned}
w_{1 ; \gamma} & =\mathcal{H}_{1}\left(u \circ f_{T ; \gamma}, \xi_{1}\right), \bar{w}_{1 ; \gamma}=w_{1 ; \gamma} \circ f_{T ; \gamma}^{-1}, \\
w_{2 ; \gamma} & =\mathcal{H}_{2}\left(u \circ f_{T ; \gamma}, \xi_{2}\right), \bar{w}_{2 ; \gamma}=w_{2 ; \gamma} \circ f_{T ; \gamma}^{-1}, \\
v_{\gamma} & =w_{1 ; \gamma}+w_{2 ; \gamma}, \bar{v}_{\gamma}=\bar{w}_{1 ; \gamma}+\bar{w}_{2 ; \gamma}, \\
z_{\gamma} & =\mathcal{L}\left(v_{\gamma}, \xi_{3}\right), \bar{z}_{\gamma}=z_{\gamma} \circ f_{T ; \gamma}^{-1}, \\
y_{\gamma} & =f \circ z_{\gamma}, \bar{y}_{\gamma}=y_{\gamma} \circ f_{T ; \gamma}^{-1}=f \circ \bar{z}_{\gamma}, \\
y_{\gamma} & =\mathcal{H}_{3}\left(v_{\gamma}, \xi_{3}\right), \\
y_{\gamma} & =\mathcal{H}\left(u \circ f_{T ; \gamma}, x_{0}\right) \text { where } x_{0}=\left(\xi_{1}, \xi_{2}, \xi_{3}\right) .
\end{aligned}
$$

Given that $\mathcal{H}_{1}$ is $\left(\{u\}, \mathbb{S}_{T ; d}^{*}, \Xi_{1}\right)$-rate independent it comes that $\mathcal{H}_{1}\left(u \circ f_{T ; \gamma}, \xi_{1}\right) \circ f_{T ; \gamma}^{-1}=$ $\mathcal{H}_{1}\left(u, \xi_{1}\right)=w_{1}$. This implies that $\left.\bar{w}_{1 ; \gamma}=w_{1}, \forall \gamma \in\right] 0, \infty\left[\right.$. Also, since $\mathcal{H}_{1}$ is confluent with respect to $\left(u, \xi_{1}, \mathbb{S}_{1 ; 1 ; d}^{*}\right)$ we have

$$
\lim _{k \rightarrow \infty}\left\|\left.\left(w_{1} \circ \tau_{k T}\right)\right|_{[0, T]}-\alpha_{u ; \mathcal{H}_{1} ; \xi_{1}}\right\|=0 .
$$

Furthermore, Equation (40) gives

$$
\lim _{(k, \gamma) \rightarrow \infty}\left(\sup _{f_{T ; \gamma} \in \mathbb{S}_{T ; \gamma ; d}^{*}}\left\|\left.\left(\bar{w}_{2 ; \gamma} \circ \tau_{k T}\right)\right|_{[0, T]}\right\|\right)=0 .
$$


On the other hand,

$$
\begin{aligned}
\dot{x}_{\gamma} & =A x_{\gamma}+B v_{\gamma}, \bar{x}_{\gamma}=x_{\gamma} \circ f_{T ; \gamma}^{-1}, \\
z_{\gamma} & =C x_{\gamma}, \bar{z}_{\gamma}=C \bar{x}_{\gamma}, \\
x_{\gamma}(0) & =\xi_{3}, \bar{x}_{\gamma}(0)=\xi_{3},
\end{aligned}
$$

so that by Proposition Appendix A.1

$$
\dot{f}_{T ; \gamma}\left(f_{T ; \gamma}^{-1}(t)\right) \dot{\bar{x}}_{\gamma}(t)=A \bar{x}_{\gamma}(t)+B \bar{v}_{\gamma}, \text { for almost all } t \in \mathbb{R}_{+} .
$$

Since $\|\overbrace{f_{T ; 1}^{-1}}^{\cdot}\| \leq d$ it comes that $\dot{f}_{T ; \gamma}\left(f_{T ; \gamma}^{-1}(t)\right)$ can be zero or undefined only on a set of measure zero. Thus

$$
\begin{aligned}
& \dot{\bar{x}}_{\gamma}(t)=\frac{1}{\dot{f}_{T ; \gamma}\left(f_{T ; \gamma}^{-1}(t)\right)}\left(A \bar{x}_{\gamma}(t)+B \bar{v}_{\gamma}\right)=\overbrace{f_{T ; \gamma}^{-1}}^{-1}(t)\left(A \bar{x}_{\gamma}(t)+B \bar{v}_{\gamma}\right), \\
& \text { for almost all } t \in \mathbb{R}_{+}, \\
\bar{x}_{\gamma}(0)= & \xi_{3} .
\end{aligned}
$$

Solving (B.15)-(B.16) we get

$$
\begin{aligned}
\bar{x}_{\gamma}(t) & =\Psi(t) \bar{x}_{\gamma}(0)+\Psi(t) \int_{0}^{t} \Psi^{-1}(\tau) b(\tau) \mathrm{d} \tau, \forall t \in \mathbb{R}_{+}, \\
\Psi(t) & =\exp (\int_{0}^{t} \overbrace{f_{T ; \gamma}^{-1}}^{\cdot}(\tau) \mathrm{d} \tau A)=\exp \left(f_{T ; \gamma}^{-1}(t) A\right), \forall t \in \mathbb{R}_{+}, \\
\Psi^{-1}(\tau) & =\exp \left(-f_{T ; \gamma}^{-1}(\tau) A\right), \forall \tau \in \mathbb{R}_{+}, \\
b(\tau) & =\overbrace{f_{T ; \gamma}^{-1}}^{\cdot}(\tau) B\left(w_{1}(\tau)+\bar{w}_{2 ; \gamma}(\tau)\right), \text { for almost all } \tau \in \mathbb{R}_{+} .
\end{aligned}
$$

Thus, $\forall t \in \mathbb{R}_{+}$we have

$$
\bar{x}_{\gamma}(t)=\exp \left(f_{T ; \gamma}^{-1}(t) A\right) \xi_{3}+\int_{0}^{t} \exp \left[\left(f_{T ; \gamma}^{-1}(t)-f_{T ; \gamma}^{-1}(\tau)\right) A\right] \overbrace{f_{T ; \gamma}^{-1}}^{\cdot}(\tau) B\left(w_{1}(\tau)+\bar{w}_{2 ; \gamma}(\tau)\right) \mathrm{d} \tau
$$

Since $A$ is stable, there exist $a \in] 0, \infty[$ and $b \in \mathbb{N} \backslash\{0\}$ that depend solely on $A$ such that

$$
\mathcal{N}_{2}\left(e^{\delta A}\right) \leq a e^{c \delta} \max _{0 \leq j \leq b-1} \frac{\delta^{j}}{j !}, \forall \delta \in \mathbb{R}_{+},
$$


where $\mathcal{N}_{2}(X)$ is the largest singular value of the matrix $X, c<0$ is the largest real part of the eigenvalues of $A$, and $\delta^{0} / 0$ ! is taken to be 1, [27, Equation (2.8)].

Recall that $w_{1}=\mathcal{H}_{1}\left(u, \xi_{1}\right) \in W^{1 ; \infty}\left(\mathbb{R}_{+}, \mathbb{R}^{m}\right)$ since $\mathcal{H}_{1}: \mathscr{U} \times \Xi_{1} \rightarrow W^{1 ; \infty}\left(\mathbb{R}_{+}, \mathbb{R}^{m}\right)$. Thus using the change of variable $\nu=f_{T ; \gamma}^{-1}(t)-f_{T ; \gamma}^{-1}(\tau)$ in (B.21) we get

$$
\begin{aligned}
\left|\bar{x}_{\gamma}(t)\right| & =\left|\exp \left(f_{T ; \gamma}^{-1}(t) A\right) \xi_{3}+\int_{0}^{f_{T ; \gamma}^{-1}(t)} e^{\nu A} B\left(w_{1}+\bar{w}_{2 ; \gamma}\right)\left[f_{T ; \gamma}\left(f_{T ; \gamma}^{-1}(t)-\nu\right)\right] \mathrm{d} \nu\right| \\
& \leq \mathcal{N}_{2}\left[\exp \left(f_{T ; \gamma}^{-1}(t) A\right)\right]\left|\xi_{3}\right|+\left(\left\|w_{1}\right\|+c_{u ; \xi_{2}}\right) \mathcal{N}_{2}(B) \int_{0}^{\infty} \mathcal{N}_{2}\left(e^{\nu A}\right) \mathrm{d} \nu, \forall \gamma \in\left[\gamma_{u ; \xi_{2}}, \infty[\right.
\end{aligned}
$$

where we have used (39). Owing to (B.22) it comes from (B.23) that

$$
\left|\bar{x}_{\gamma}(t)\right| \leq R, \forall \gamma \in\left[\gamma_{u ; \xi_{2}}, \infty\left[, \forall t \in \mathbb{R}_{+},\right.\right.
$$

where $R \in \mathbb{R}_{+}$is a constant independent of $\gamma$ and of $f_{T ; \gamma}$.

Define the following constants: $c_{3}=a \mathcal{N}_{2}(B) \int_{0}^{\infty} e^{c \nu} \max _{0 \leq j \leq b-1} \frac{\nu^{j}}{j !} \mathrm{d} \nu, c_{5}=\left(2+c_{3}\right) \mathcal{N}_{2}(C)$, $c_{6}=\mathcal{N}_{2}(G)+c_{5}, k^{*}=1+\left\|G \alpha_{u ; \mathcal{H}_{1} ; \xi_{1}}\right\|$.

Since $f$ is continuous, it is uniformly continuous on the closed ball $\overline{\mathscr{B}}\left(\mathbf{0}_{r}, k^{*}\right)$ centered on the origin of coordinates $\mathbf{0}_{r} \in \mathbb{R}^{r}$ with radius $k^{*}$.

Take $\epsilon \in] 0, \infty\left[\right.$. Then, $\left.\left.\exists \delta_{\epsilon} \in\right] 0,1\right]$ such that $|f(\boldsymbol{x})-f(\boldsymbol{y})| \leq \epsilon, \forall \boldsymbol{x}, \boldsymbol{y} \in \overline{\mathscr{B}}\left(\mathbf{0}_{r}, k^{*}\right)$ with $|\boldsymbol{x}-\boldsymbol{y}| \leq \delta_{\epsilon}$.

By (B.9) $\exists k_{\epsilon} \in \mathbb{N} \backslash\{0\}$ and $\exists \gamma_{\epsilon} \in\left[\gamma_{u ; \xi_{2}}, \infty[\right.$ such that

$$
\left\|\left.\left(\bar{w}_{2 ; \gamma} \circ \tau_{k T}\right)\right|_{[0, T]}\right\| \leq \epsilon_{1}=\frac{\delta_{\epsilon}}{1+c_{6}}, \forall k \in \llbracket k_{\epsilon}, \infty \llbracket, \forall \gamma \in\left[\gamma_{\epsilon}, \infty[.\right.
$$

Define $t_{\epsilon}=k_{\epsilon} T$. Then it comes from (B.21) that $\forall t \in\left[t_{\epsilon}, \infty[\right.$ we have

$$
\begin{aligned}
\bar{x}_{\gamma}(t)= & \exp \left[\left(f_{T ; \gamma}^{-1}(t)-f_{T ; \gamma}^{-1}\left(t_{\epsilon}\right)\right) A\right] \bar{x}_{\gamma}\left(t_{\epsilon}\right) \\
& +\int_{t_{\epsilon}}^{t} \exp \left[\left(f_{T ; \gamma}^{-1}(t)-f_{T ; \gamma}^{-1}(\tau)\right) A\right] \overbrace{f_{T ; \gamma}^{-1}}^{\cdot}(\tau) B\left(w_{1}(\tau)+\bar{w}_{2 ; \gamma}(\tau)\right) \mathrm{d} \tau .
\end{aligned}
$$

Integrating by parts we obtain

$$
\begin{aligned}
& \int_{t_{\epsilon}}^{t}-A \exp \left[\left(f_{T ; \gamma}^{-1}(t)-f_{T ; \gamma}^{-1}(\tau)\right) A\right] \overbrace{f_{T ; \gamma}^{-1}}^{\cdot}(\tau) B w_{1}(\tau) \mathrm{d} \tau=B w_{1}(t) \\
& -\exp \left[\left(f_{T ; \gamma}^{-1}(t)-f_{T ; \gamma}^{-1}\left(t_{\epsilon}\right)\right) A\right] B w_{1}\left(t_{\epsilon}\right)-\int_{t_{\epsilon}}^{t} \exp \left[\left(f_{T ; \gamma}^{-1}(t)-f_{T ; \gamma}^{-1}(\tau)\right) A\right] B \dot{w}_{1}(\tau) \mathrm{d} \tau .
\end{aligned}
$$


We have

$$
\begin{aligned}
& \left|\int_{t_{\epsilon}}^{t} \exp \left[\left(f_{T ; \gamma}^{-1}(t)-f_{T ; \gamma}^{-1}(\tau)\right) A\right] B \dot{w}_{1}(\tau) \mathrm{d} \tau\right| \\
& \leq a\left\|\dot{w}_{1}\right\| \mathcal{N}_{2}(B) \int_{t_{\epsilon}}^{t} \exp \left[c\left(f_{T ; \gamma}^{-1}(t)-f_{T ; \gamma}^{-1}(\tau)\right)\right] \max _{0 \leq j \leq b-1} \frac{\left(f_{T ; \gamma}^{-1}(t)-f_{T ; \gamma}^{-1}(\tau)\right)^{j}}{j !} \mathrm{d} \tau \\
& =a\left\|\dot{w}_{1}\right\| \mathcal{N}_{2}(B) \int_{0}^{f_{T ; \gamma}^{-1}(t)-f_{T ; \gamma}^{-1}\left(t_{\epsilon}\right)} \frac{\dot{f}_{T ; 1}\left(f_{T ; 1}^{-1}(\tau)\right)}{\gamma} e^{c \tau} \max _{0 \leq j \leq b-1} \frac{\tau^{j}}{j !} \mathrm{d} \tau \\
& \leq \frac{1}{\gamma} a\left\|\dot{w}_{1}\right\| d \mathcal{N}_{2}(B) \int_{0}^{\infty} e^{c \tau} \max _{0 \leq j \leq b-1} \frac{\tau^{j}}{j !} \mathrm{d} \tau=\frac{c_{1}}{\gamma},
\end{aligned}
$$

where $c_{1}=a\left\|\dot{w}_{1}\right\| d \mathcal{N}_{2}(B) \int_{0}^{\infty} e^{c \tau} \max _{0 \leq j \leq b-1} \frac{\tau^{j}}{j !} \mathrm{d} \tau$.

On the other hand,

$$
\begin{aligned}
& \left|\exp \left[\left(f_{T ; \gamma}^{-1}(t)-f_{T ; \gamma}^{-1}\left(t_{\epsilon}\right)\right) A\right]\left(\bar{x}_{\gamma}\left(t_{\epsilon}\right)+A^{-1} B w_{1}\left(t_{\epsilon}\right)\right)\right| \\
& \leq \underbrace{a\left(R+\left\|w_{1}\right\| \mathcal{N}_{2}\left(A^{-1} B\right)\right)}_{=c_{2}} \exp \left[c \gamma\left(f_{T ; 1}^{-1}(t)-f_{T ; 1}^{-1}\left(t_{\epsilon}\right)\right)\right] \max _{0 \leq j \leq b-1} \frac{\left[\gamma\left(f_{T ; 1}^{-1}(t)-f_{T ; 1}^{-1}\left(t_{\epsilon}\right)\right)\right]^{j}}{j !} .
\end{aligned}
$$

The only global maximum of the function $\kappa: \mathbb{R}_{+} \rightarrow \mathbb{R}_{+}$defined by $\kappa(\sigma)=e^{c \gamma \sigma}(\gamma \sigma)^{j}$ is attained at $\sigma=0$ for $j=0$, at $\sigma=-\frac{1}{c \gamma}$ for $j=1$ and at $\sigma=-\frac{j}{\gamma^{2} c}$ for $j>1$. It can be checked that $\forall \gamma \geq b$ the function $\kappa$ is strictly decreasing on the interval $\left[-\frac{1}{c b}=\sigma_{0}, \infty[\right.$ for any value of $0 \leq j \leq b-1$. Thus

$$
e^{c \gamma \sigma} \max _{0 \leq j \leq b-1} \frac{(\gamma \sigma)^{j}}{j !} \leq \max _{0 \leq j \leq b-1} \frac{e^{c \gamma \sigma_{0}}\left(\gamma \sigma_{0}\right)^{j}}{j !}, \forall \sigma \in\left[\sigma_{0}, \infty[, \forall \gamma \geq b .\right.
$$

Since $\left\|\dot{f}_{T ; 1}\right\| \leq d$ it comes that $\forall t \in\left[f_{T ; 1}^{-1}\left(t_{\epsilon}\right), \infty\left[, f_{T ; 1}(t) \leq t_{\epsilon}+d\left(t-f_{T ; 1}^{-1}\left(t_{\epsilon}\right)\right)\right.\right.$. For $t=\sigma_{0}+f_{T ; 1}^{-1}\left(t_{\epsilon}\right)$ we obtain $f_{T ; 1}\left(\sigma_{0}+f_{T ; 1}^{-1}\left(t_{\epsilon}\right)\right) \leq t_{\epsilon}+d \sigma_{0}$. Thus $\forall t \in\left[t_{\epsilon}+d \sigma_{0}, \infty\left[, f_{T ; 1}^{-1}(t) \geq\right.\right.$ $f_{T ; 1}^{-1}\left(t_{\epsilon}+d \sigma_{0}\right) \geq \sigma_{0}+f_{T ; 1}^{-1}\left(t_{\epsilon}\right)$ so that $f_{T ; 1}^{-1}(t)-f_{T ; 1}^{-1}\left(t_{\epsilon}\right) \geq \sigma_{0}$. Then Inequality (B.30) leads to

$$
\begin{aligned}
& \exp \left[c \gamma\left(f_{T ; 1}^{-1}(t)-f_{T ; 1}^{-1}\left(t_{\epsilon}\right)\right)\right] \max _{0 \leq j \leq b-1} \frac{\left[\gamma\left(f_{T ; 1}^{-1}(t)-f_{T ; 1}^{-1}\left(t_{\epsilon}\right)\right)\right]^{j}}{j !} \\
& \leq \max _{0 \leq j \leq b-1} \frac{e^{c \gamma \sigma_{0}}\left(\gamma \sigma_{0}\right)^{j}}{j !}, \forall t \in\left[t_{\epsilon}+d \sigma_{0}, \infty[, \forall \gamma \geq b .\right.
\end{aligned}
$$


Combining Equations (B.31) and (B.29) it follows that

$$
\begin{aligned}
& \left|\exp \left[\left(f_{T ; \gamma}^{-1}(t)-f_{T ; \gamma}^{-1}\left(t_{\epsilon}\right)\right) A\right]\left(\bar{x}_{\gamma}\left(t_{\epsilon}\right)+A^{-1} B w_{1}\left(t_{\epsilon}\right)\right)\right| \\
& \leq c_{2} \max _{0 \leq j \leq b-1} \frac{e^{c \gamma \sigma_{0}}\left(\gamma \sigma_{0}\right)^{j}}{j !}, \forall t \in\left[t_{\epsilon}+d \sigma_{0}, \infty[, \forall \gamma \geq b .\right.
\end{aligned}
$$

Also, using the change of variable $\nu=f_{T ; \gamma}^{-1}(t)-f_{T ; \gamma}^{-1}(\tau)$ we get

$$
\begin{aligned}
& \int_{t_{\epsilon}}^{t} \exp \left[\left(f_{T ; \gamma}^{-1}(t)-f_{T ; \gamma}^{-1}(\tau)\right) A\right] \overbrace{f_{T ; \gamma}^{-1}}^{\cdot}(\tau) B \bar{w}_{2 ; \gamma}(\tau) \mathrm{d} \tau \\
& =\int_{0}^{f_{T ; \gamma}^{-1}(t)-f_{T ; \gamma}^{-1}\left(t_{\epsilon}\right)} \exp (\nu A) B \bar{w}_{2 ; \gamma}\left[f_{T ; \gamma}\left(f_{T ; \gamma}^{-1}(t)-\nu\right)\right] \mathrm{d} \nu .
\end{aligned}
$$

Using (B.25) it follows that

$$
\begin{aligned}
& \left|\int_{0}^{f_{T ; \gamma}^{-1}(t)-f_{T ; \gamma}^{-1}\left(t_{\epsilon}\right)} \exp (\nu A) B \bar{w}_{2 ; \gamma}\left[f_{T ; \gamma}\left(f_{T ; \gamma}^{-1}(t)-\nu\right)\right] \mathrm{d} \nu\right| \\
& \leq \epsilon_{1} a \mathcal{N}_{2}(B) \int_{0}^{\infty} e^{c \nu} \max _{0 \leq j \leq b-1} \frac{\nu^{j}}{j !} \mathrm{d} \nu=\epsilon_{1} c_{3}, \forall \gamma \in\left[\gamma_{\epsilon}, \infty\left[, \forall t \in\left[t_{\epsilon}, \infty[\right.\right.\right.
\end{aligned}
$$

where $c_{3}=a \mathcal{N}_{2}(B) \int_{0}^{\infty} e^{c \nu} \max _{0 \leq j \leq b-1} \frac{\nu^{j}}{j !} \mathrm{d} \nu$.

Combining Equations (B.33)-(B.34), (B.26)-(B.28) and (B.32) it comes that

$$
\begin{aligned}
& \left|\bar{x}_{\gamma}(t)+A^{-1} B w_{1}(t)\right| \leq \frac{c_{4}}{\gamma}+c_{2} \max _{0 \leq j \leq b-1} \frac{e^{c \gamma \sigma_{0}}\left(\gamma \sigma_{0}\right)^{j}}{j !}+\epsilon_{1} c_{3}, \forall t \in\left[t_{\epsilon}+d \sigma_{0}, \infty[,\right. \\
& \forall \gamma \in\left[\gamma_{\epsilon ; 1}, \infty[\right.
\end{aligned}
$$

where $c_{4}=c_{1} \mathcal{N}_{2}\left(A^{-1}\right)$ and $\gamma_{\epsilon ; 1}=\max \left(\gamma_{\epsilon}, b\right)$.

We have

$$
\lim _{\gamma \rightarrow \infty} \max _{0 \leq j \leq b-1} \frac{e^{c \gamma \sigma_{0}}\left(\gamma \sigma_{0}\right)^{j}}{j !}=0
$$

so that $\left.\exists \gamma_{0} \in\right] 0, \infty\left[\right.$ such that $c_{2} \max _{0 \leq j \leq b-1} \frac{e^{c \gamma \sigma_{0}}\left(\gamma \sigma_{0}\right)^{j}}{j !} \leq \epsilon_{1}, \forall \gamma \in\left[\gamma_{0}, \infty[\right.$. Define $\gamma_{\epsilon ; 2}=\max \left(\gamma_{\epsilon ; 1}, \gamma_{0}, c_{4} / \epsilon_{1}\right), k_{\epsilon ; 1}=\left\lceil\frac{t_{\epsilon}+d \sigma_{0}}{T}\right\rceil$, then (B.35) gives

$$
\left\|\left.\left(\left(C \bar{x}_{\gamma}+C A^{-1} B w_{1}\right) \circ \tau_{k T}\right)\right|_{[0, T]}\right\| \leq c_{5} \epsilon_{1}, \forall \gamma \in\left[\gamma_{\epsilon ; 2}, \infty\left[, \forall k \in \llbracket k_{\epsilon ; 1}, \infty \llbracket,\right.\right.
$$

where $c_{5}=\left(2+c_{3}\right) \mathcal{N}_{2}(C)$. 
On the other hand, (B.8) ensures that $\exists k_{\epsilon ; 2} \in \llbracket k_{\epsilon ; 1}, \infty \llbracket$ such that

$$
\left\|\left.\left(w_{1} \circ \tau_{k T}\right)\right|_{[0, T]}-\alpha_{u ; \mathcal{H}_{1} ; \xi_{1}}\right\| \leq \epsilon_{1}, \forall k \in \llbracket k_{\epsilon ; 2}, \infty \llbracket .
$$

Combining Equations (B.36)-(B.37) and (B.11) we obtain

$$
\left\|\left.\left(\bar{z}_{\gamma} \circ \tau_{k T}\right)\right|_{[0, T]}-G \alpha_{u ; \mathcal{H}_{1} ; \xi_{1}}\right\| \leq c_{6} \epsilon_{1}<\delta_{\epsilon}, \forall \gamma \in\left[\gamma_{\epsilon ; 2}, \infty\left[, \forall k \in \llbracket k_{\epsilon ; 2}, \infty \llbracket,\right.\right.
$$

where $c_{6}=\mathcal{N}_{2}(G)+c_{5}$.

On the other hand,

$$
\begin{aligned}
\left|\bar{y}_{\gamma}(t)-f\left(G \alpha_{u ; \mathcal{H}_{1} ; \xi_{1}}(t)\right)\right| & =\left|f\left(\bar{z}_{\gamma}(t)\right)-f\left(G \alpha_{u ; \mathcal{H}_{1} ; \xi_{1}}(t)\right)\right| \\
& =\left|f\left(G \alpha_{u ; \mathcal{H}_{1} ; \xi_{1}}(t)+\kappa_{\gamma}(t)\right)-f\left(G \alpha_{u ; \mathcal{H}_{1} ; \xi_{1}}(t)\right)\right|
\end{aligned}
$$

where $\kappa_{\gamma}(t)=\bar{z}_{\gamma}(t)-G \alpha_{u ; \mathcal{H}_{1} ; \xi_{1}}(t)$. Note that

$$
G \alpha_{u ; \mathcal{H}_{1} ; \xi_{1}}(t)+\kappa_{\gamma}(t) \in \overline{\mathscr{B}}\left(\mathbf{0}_{r}, k^{*}\right), \forall \gamma \in\left[\gamma_{\epsilon ; 2}, \infty\left[, \forall t \in\left[k_{\epsilon ; 2} T, \infty[\right.\right.\right.
$$

owing to (B.38), and that $G \alpha_{u ; \mathcal{H}_{1} ; \xi_{1}}(t) \in \overline{\mathscr{B}}\left(\mathbf{0}_{r}, k^{*}\right), \forall t \in \mathbb{R}_{+}$. Thus

$$
\left|f\left(G \alpha_{u ; \mathcal{H}_{1} ; \xi_{1}}(t)+\kappa_{\gamma}(t)\right)-f\left(G \alpha_{u ; \mathcal{H}_{1} ; \xi_{1}}(t)\right)\right| \leq \epsilon, \forall \gamma \in\left[\gamma_{\epsilon ; 2}, \infty\left[, \forall t \in\left[k_{\epsilon ; 2} T, \infty[.\right.\right.\right.
$$

We have thus shown that $\forall \epsilon \in] 0, \infty\left[, \exists \gamma_{\epsilon ; 2} \in\right] 0, \infty\left[, \exists k_{\epsilon ; 2} \in \mathbb{N}\right.$ such that

$$
\left\|\left.\left(\bar{y}_{\gamma} \circ \tau_{k T}\right)\right|_{[0, T]}-f \circ\left(G \alpha_{u ; \mathcal{H}_{1} ; \xi_{1}}\right)\right\| \leq \epsilon, \forall \gamma \in\left[\gamma_{\epsilon ; 2}, \infty\left[, \forall k \in \llbracket k_{\epsilon ; 2}, \infty \llbracket,\right.\right.
$$

which ends the proof.

\section{Acknowledgment}

Funding: This study was funded by the Spanish Ministry of Economy, Industry and Competitiveness (grant number DPI2016-77407-P (AEI/FEDER, UE)).

\section{Compliance with Ethical Standards}

Conflict of Interest: The author declares that they have no conflict of interest. 


\section{References}

[1] O. Aljanaideh, D. Habineza, M. Rakotondrabe, M. AlJanaideh, Experimental comparison of rate-dependent hysteresis models in characterizing and compensating hysteresis of piezoelectric tube actuators, Physica B. 486 (2016) 64-68.

[2] P. Albertos, A. Sala, Multivariable Control System, Springer-Verlag, London, 2004.

[3] G. Bertotti, I. Mayergoyz, ed., The Science of Hysteresis, 3-volume set, Elsevier, Academic Press, Oxford, UK, 2006.

[4] M. Brokate, J. Sprekels, Hysteresis and Phase Transitions, Springer-Verlag, New York, USA, 1996.

[5] R. Bouc, Modèle mathématique d'hystérésis, Acustica. 21 (1971) 16-25.

[6] E. Della Torre, Magnetic Hysteresis, IEEE Press, Piscataway, NJ, USA, 1999.

[7] P. Duhem, Sur les déformations permanentes et l'hystérésis. Premier Mémoire, tome LIV, Mémoires couronnés et Mémoires des savants étrangers, l'Académie royale des sciences, des lettres et des beaux-arts de Belgique, 1896.

[8] P. Duhem, Les modifications permanentes du soufre. Deuxième Mémoire, tome LIV, Mémoires couronnés et Mémoires des savants étrangers, l'Académie royale des sciences, des lettres et des beaux-arts de Belgique, 1896.

[9] P. Duhem, Théorie générale des déformations permanentes. Troisième Mémoire, tome LIV, Mémoires couronnés et Mémoires des savants étrangers, l'Académie royale des sciences, des lettres et des beaux-arts de Belgique, 1896.

[10] P. Duhem, Étude de divers systèmes dépendant d'une seule variable. Quatrième Mémoire, tome LVI, Mémoires couronnés et Mémoires des savants étrangers, l'Académie royale des sciences, des lettres et des beaux-arts de Belgique, 1898.

[11] P. Duhem, Étude de divers systèmes dépendant de deux variables. Cinquième Mémoire, tome LVI, Mémoires couronnés et Mémoires des savants étrangers, l'Académie royale des sciences, des lettres et des beaux-arts de Belgique, 1898.

[12] P. Duhem, L'inégalité de Clausius et l'hystérésis. Sixième Mémoire, tome LXII, Mémoires couronnés et autres Mémoires, l’Académie royale de Belgique, 1902. 
[13] P. Duhem, Hystérésis et viscosité. Septième Mémoire, tome LXII, Mémoires couronnés et autres Mémoires, l'Académie royale de Belgique, 1902.

[14] J.A. Ewing, On the production of transient electric currents in iron and steel conductors by twisting them when magnetised or by magnetising them when twisted, Proc. R. Soc. Lond. 33 (1881) 21-23.

[15] I. García-Baños, F. Ikhouane, A new method for the identification of the parameters of the Dahl model, J. Phys.: Conf. Ser. 744 (2016) 012195.

[16] J. Gan, X. Zhang, Modeling of rate-dependent hysteresis in piezoelectric actuators based on a modified Prandtl-Ishlinskii model, Int. J. Appl. Electromag. Mech. 49 (2015) 557-565.

[17] F. Ikhouane, Characterization of hysteresis processes, Math. Control Signals Syst. 25 (2013), 294-310.

[18] F. Ikhouane, A survey of the hysteretic Duhem model. Arch. Comput. Meth. Eng. 25(4) (2018) 965-1002.

[19] F. Ikhouane, F. Giri, A unified approach for the parametric identification of SISO/MIMO Wiener and Hammerstein systems, J. Franklin. Inst. 351 (2014) 17171727.

[20] F. Ikhouane, J. Rodellar, Systems with Hysteresis: Analysis, Identification and Control using the Bouc-Wen model, John Wiley \& Sons, The Atrium, Southern Gate, Chichester, England, 2007.

[21] M.A. Krasnosel'skiǐ, A.V. Pokrovskǐ̌, Systems with Hysteresis, Springer-Verlag, 1989.

[22] J.W. Macki, P. Nistri, P. Zecca, Mathematical models for hysteresis, SIAM Review. 35 (1993) 94-123.

[23] I. Mayergoyz, Mathematical Models of Hysteresis, Elsevier Series in Electromagnetism, Elsevier, 2003.

[24] G. Leoni, A First Course in Sobolev Spaces, The American Mathematical Society, USA, 2009.

[25] J. Oh, B. Drinčić, D.S. Bernstein, Nonlinear feedback models of hysteresis, IEEE Contr. Syst. Mag. 29 (2009) 100-119. 
[26] J. Oh and D.S. Bernstein, Semilinear Duhem model for rate-independent and ratedependent hysteresis. IEEE Trans. Aut. Contr. 50 (2005) 631-645.

[27] C. Van Loan, The sensitivity of the matrix exponential, SIAM. J. Numer. Anal. 14 (1977) 971-981.

[28] A. Visintin, Differential Models of Hysteresis, Springer-Verlag, Berlin, Heidelberg, 1994.

[29] A. Visintin. Mathematical models of hysteresis. In: G. Bertotti and I. Mayergoyz, ed. The Science of Hysteresis, Academic Press, volume I, chapter 1, 2005.

[30] M-Ju Yang, C-X Li, G-Y Gu, L-M Zhu, A modified Prandtl-Ishlinskii model for ratedependent hysteresis nonlinearity using an mth-power velocity damping mechanism, Int. J. Adv. Robot. Syst. 11 (2014), Article Number: 163.

[31] E.H. Yen, H.R. Van Der Vaart, On measurable functions, continuous functions and some related concepts, Amer. Math. Month. 73 (1966) 991-993.

[32] Z. Zhang, Y. Ma, Modeling of rate-dependent hysteresis using a GPO-based adaptive filter, Sensors, 16 (2016) DOI: 10.3390/s16020205. 